\title{
Axisymmetric travelling waves in annular sliding Couette flow at finite and asymptotically large Reynolds number
}

\author{
By K. DEGUCHI AND A. G. WALTON ${ }^{2}$ \\ ${ }^{1}$ Department of Aeronautics and Astronautics, Graduate School of Engineering, \\ Kyoto University, Kyoto, 606-8501, JAPAN \\ 2 Department of Mathematics, Imperial College London, \\ South Kensington Campus, London SW7 2AZ, UK
}

The relationship between numerical finite-amplitude equilibrium solutions of the full Navier-Stokes equations and nonlinear solutions arising from a high Reynolds number asymptotic analysis is discussed for a Tollmien-Schlichting wave type two-dimensional vortical flow structure. The specific flow chosen for this purpose is that which arises from the mutual axial sliding of co-axial cylinders for which nonlinear axisymmetric travellingwave solutions have been discovered recently by Deguchi \& Nagata (J. Fluid Mech., vol. 678,2011 , pp. 156-178). We continue this solution branch to a Reynolds number $R=10^{8}$ and confirm that the behaviour of its so-called lower branch solutions, which typically produce a smaller modification to the laminar state than the other solution branches, quantitatively agrees with the axisymmetric asymptotic theory developed in this paper. We further find that this asymptotic structure breaks down when the disturbance wavelength is comparable with $R$. The new structure which replaces it is investigated and the governing equations are derived and solved. The flow visualization of the resultant solutions reveals that they possess a streamwise localized structure, with the trend agreeing qualitatively with full Navier-Stokes solutions for relatively long wavelength disturbances.

\section{Introduction}

It is known that laminar-turbulent transition in shear flows involves two types of distinct vortical structure. The first of these structures arises by consideration of the linear stability of basic solutions to the Navier-Stokes equations. As Tollmien (1929) and Schlichting (1933) found for boundary-layer flow, an infinitesimally small growing mode typically has a two-dimensional orthogonally-aligned roll pattern. In contrast, experimental results and direct Navier-Stokes simulations (e.g. Davies \& White 1928, Nishioka et al. 1975 and Lemoult et al. 2012 for experiments, Orszag \& Kells 1980, Henningson et al. 1987 and Tsukahara et al. 2005 for simulations) indicate that shear flows can lose stability to nonlinear three-dimensional streamwise roll structures at much lower Reynolds numbers than those associated with the two-dimensional linear disturbances mentioned above. In addition, there exists anomalistic flows which do not exhibit any linear instability: for example plane Couette flow, pipe flow and square duct flow.

This apparent conflict between theory and experiment has been resolved by the computation of three-dimensional finite-amplitude solutions of the Navier-Stokes equations (e.g. Nagata 1990 and Clever \& Busse 1997 for plane Couette flow, Faisst \& Eckhardt 
2003 and Wedin \& Kerswell 2004 for pipe flow, Waleffe 1998 for plane Poiseuille flow). These nonlinear states possess a roll-streak coherent structure and are disconnected from any linear instability, appearing abruptly by means of a saddle-node bifurcation at the value of Reynolds number, $R$, at which the corresponding flow dynamics first begin to show instability according to experiments or unsteady Navier-Stokes computations. Controlled Navier-Stokes simulations (e.g. Itano \& Toh 2001 and Skufca, Yorke \& Eckhardt 2006) show that these solutions are situated at the edge of the laminar-turbulent boundary and play a 'gatekeeper' role in the transition dynamics. However, it should be noted that these investigations are undertaken far away from the linearly unstable parameter region. Therefore, such a three-dimensional instability could 'pass the torch' to a Tollmien-Schlichting type disturbance as the critical Reynolds number for linear stability is approached. Consequently, two-dimensional finite-amplitude solutions bifurcating from the linear neutral point could play a crucial role when we consider laminar-turbulent transition control at high Reynolds number.

One of the primary aims of this paper is to attempt to describe the structure of the nonlinear travelling-wave solutions using high Reynolds number asymptotic theory. The theory that we outline here in section 5 involves the presence of a strongly nonlinear equilibrium critical layer in which viscosity only plays a higher order role. The dominant physical balances are similar to those proposed in studies by Benney \& Bergeron (1969) and Davis (1969), and the properties of such critical layers were investigated further by Haberman (1972), Brown \& Stewartson (1978), Smith \& Bodonyi (1982a,b) and Bodonyi, Smith \& Gajjar (1983). One of the key features which sustains the asymptotic structure is the jump in vorticity across the layer, which acts as a forcing of the mean-flow distortion in the bulk of the flow outside the critical layer. Although the properties of these critical layers and the associated surrounding flow have been known for some time, the delicate structure of the overall solution, with its asymptotically thin internal and boundary layers, has proved difficult to detect in full Navier-Stokes computations thus far. In this paper, by explicitly seeking nonlinear travelling-wave solutions of the NavierStokes equations, and using recent advances in computing power to allow us to compute solutions at Reynolds numbers in excess of $10^{8}$, we are able, for the first time, to quantitatively compare the flow structures present in full Navier-Stokes computations with those resulting from an asymptotic analysis. This comparison of full finite-amplitude solutions and asymptotic analysis is motivated in part by the work of Hall \& Sherwin (2010), who recently successfully linked the asymptotic behaviour of the three-dimensional solutions described above to the high Reynolds number vortex-wave interaction theory developed by Hall \& Smith (1991). In contrast to vortex-wave interaction theory, where the flow must be necessarily three-dimensional, the asymptotic theory described in this paper is derived under the assumption of two-dimensionality, so that the disturbances resemble Tollmien-Schlichting waves, i.e. they are invariant under any translation in one specific direction.

If we consider a one-parameter family of flows which exhibit linear instability below a critical value of that parameter, it is often found that, as the critical value is approached, the linearly unstable region recedes to $R=\infty$ with the wavenumber scaling as $O\left(R^{-1}\right)$, (e.g. see Cowley \& Smith (1985) for plane Couette-Poiseuille flow and Gittler (1993) for the corresponding generalization to a cylindrical geometry). One of our interests in this paper concerns the fully nonlinear version of this long-wave cut-off. We find that the two-dimensional asymptotic structure is broken at the long-wave limit and a new structure emerges. In the new regime all streamwise Fourier modes become leading order and we will see that the solution develops a strongly streamwise-localized structure. 
Corresponding work carried out for vortex-wave interaction theory has been recently described in Deguchi, Hall \& Walton (2013).

In this study, the specific flow we consider is that where the fluid motion is produced by the mutual axial sliding motion of co-axial cylinders of circular cross-section, and the fluid occupies the annular region between the cylinders. We refer to this flow as annular sliding Couette flow, and the basic velocity profile is given in equation (2.2) below. There are three reasons for the selection of annular sliding Couette flow: (i) there exists an axisymmetric linear instability if the radius ratio is less than 0.1415 (Gittler 1993); (ii) axisymmetric finite-amplitude travelling-wave solutions bifurcating from this linear instability have been found at very high Reynolds number by Deguchi \& Nagata (2011, hereinafter referred to as DN11); (iii) asymptotic theory has already been developed for annular Couette-Poiseuille flow, which is the non-zero axial pressure gradient case of annular sliding Couette flow, by Walton (2003, 2004 and 2005). In addition, comparisons can be made with plane Couette flow in the limit as the radius ratio tends to unity. The flow has many industrial and medical applications especially when extended to annular Couette-Poiseuille flow (for details see the introduction of DN11) while the zero pressure gradient state has some relevance to the boundary layer flow along a cylinder (e.g. Tutty 2008 and Cipolla \& Keith 2003): see section 6 of Gittler (1993). Despite these important applications, experimental investigations of annular flow with a sliding inner core can only found in Shands et al. (1980) and Frei et al. (2000).

Although our study concentrates on annular sliding Couette flow, the story presented in the rest of the paper would proceed along similar lines for any Tollmien-Schlichting wave type two-dimensional instability, e.g. the two-dimensional finite-amplitude solutions for plane Couette-Poiseuille flow (Cherhabili \& Ehrenstein 1995) and annular CouettePoiseuille flow (Wong \& Walton 2012).

In the next section we begin by formulating annular sliding Couette flow mathematically. Section 3 is devoted to an energy analysis of the flow which proves useful when checking the reliability of our numerically-generated solution branches. The numerical method used to obtain axisymmetric travelling waves at finite Reynolds number is presented in section 4 and the corresponding high Reynolds number asymptotic theory is given in section 5 . The results obtained using these two approaches are compared quantitatively in section 6 , while the small wavenumber limit of our solutions is investigated in section 7 . Finally, in section 8 , we summarize our findings and draw some conclusions.

\section{Formulation of the problem}

We consider an incompressible viscous fluid flow in the annular region between infinitely long co-axial cylinders. Annular sliding Couette flow is assumed throughout this paper, i.e. the flow is driven by the mutual motion of the cylinders with no axial pressure gradient applied. We will assume that the motion is axisymmetric and axially periodic. The length, velocity and density scales are chosen so that the half-gap, inner cylinder velocity and density are normalized to unity. There are then three parameters in the problem: the radius ratio $\eta<1$, Reynolds number $R$ and axial wavenumber $\alpha$. Keeping the outer cylinder at rest, the Reynolds number can be defined as the half-gap multiplied by the inner cylinder velocity and divided by the kinematic viscosity of the fluid. As a consequence, the flow is governed by the non-dimensional Navier-Stokes equations in a periodic annular domain spanned by the axial and radial coordinates

$$
x \in[0,2 \pi / \alpha], \quad r \in\left[r_{a}, r_{b}\right] \equiv[2 \eta /(1-\eta), 2 /(1-\eta)] .
$$


We define the unit vectors in the axial and radial directions as $\mathbf{e}_{x}$ and $\mathbf{e}_{r}$ respectively. The total velocities are written as $\left(U_{B}(r)+u(t, x, r)\right) \mathbf{e}_{x}+v(t, x, r) \mathbf{e}_{r}$ where the basic flow $U_{B}(r)$ is the exact Navier-Stokes solution

$$
U_{B}(r)=\frac{\ln \left(r / r_{b}\right)}{\ln \eta}
$$

The equations for the velocity disturbance $\mathbf{u}=u \mathbf{e}_{x}+v \mathbf{e}_{r}$ and pressure disturbance $p$ to the basic flow are

$$
\begin{aligned}
u_{x}+v_{r}+r^{-1} v & =0, \\
u_{t}+U_{B} u_{x}+v U_{B}^{\prime}+\mathbf{u} \cdot \nabla u & =-p_{x}+R^{-1} \nabla^{2} u, \\
v_{t}+U_{B} v_{x}+\mathbf{u} \cdot \nabla v & =-p_{r}+R^{-1}\left(\nabla^{2} v-r^{-2} v\right),
\end{aligned}
$$

together with the no-slip boundary conditions

$$
u=v=0 \text { at } r=r_{a} \text { and } r=r_{b} .
$$

Here we use the notation

$$
\nabla=\mathbf{e}_{r} \partial_{r}+\mathbf{e}_{x} \partial_{x}, \quad \nabla^{2}=\partial_{r r}^{2}+r^{-1} \partial_{r}+\partial_{x x}^{2}
$$

and a prime denotes a derivative with respect to $r$.

\section{Energy analysis}

In this section we employ standard techniques of energy analysis (e.g. see Joseph \& Carmi 1969) to compute an energy Reynolds number $R_{E}$ for annular sliding Couette flow. This is a value of Reynolds number below which it is guaranteed that all disturbances to the basic flow decay monotonically in time, and it therefore represents a lower bound on $R$, below which finite-amplitude travelling waves cannot exist. Often, of course, this bound proves to be extremely pessimistic in practice; however it still provides a useful partial check on the validity of the travelling-wave solutions discussed in this paper and reduces the parameter space that needs to be investigated.

We begin by defining the kinetic energy of the disturbance flow as

$$
E \equiv \frac{1}{2}\left\langle u^{2}+v^{2}\right\rangle
$$

where the volumetric integrating operator takes the form

$$
\langle *\rangle \equiv \int_{0}^{2 \pi / \alpha} \int_{r_{a}}^{r_{b}} * r d r d x .
$$

The development of the energy can be found by considering $\langle u(2.4)+v(2.5)\rangle$. This leads to the exact result

where

$$
\frac{d E}{d t}=\frac{I_{D}}{R}(m-1)
$$

$$
I_{D} \equiv\left\langle|\nabla u|^{2}+|\nabla v|^{2}+r^{-2} v^{2}\right\rangle \geq 0, m \equiv R \frac{I_{A}}{I_{D}}, I_{A} \equiv-\left\langle u v U_{B}^{\prime}\right\rangle .
$$

It follows that we need $\grave{m}=\max _{\mathbf{u}} m>1$ in order for the system to experience some energy growth. Suppose that the solution which realizes $\grave{m}$ is $[\grave{u}, \grave{v}]$. We then consider all solutions in the form $[u, v]=[\grave{u}, \grave{v}]+\delta\left[\grave{u}, \grave{v}^{\prime}\right]$. By definition, $\grave{m}=\left.m\right|_{\delta=0}$ and $\left.\frac{\partial m}{\partial \delta}\right|_{\delta=0}=0$. 
Using these relations and the definition of $m$, we obtain

$$
\left.\grave{m} \frac{\partial I_{D}}{\partial \delta}\right|_{\delta=0}-\left.R \frac{\partial I_{A}}{\partial \delta}\right|_{\delta=0}=\langle\dot{\mathbf{u}} \cdot \boldsymbol{\Phi}\rangle=0
$$

where

$$
\boldsymbol{\Phi}=\left(-2 \grave{m} \nabla^{2} \grave{u}+R \grave{v} U_{B}^{\prime}\right) \mathbf{e}_{x}+\left(-2 \grave{m}\left(\nabla^{2} \grave{v}-r^{-2} \grave{v}\right)+R \grave{u} U_{B}^{\prime}\right) \mathbf{e}_{r} .
$$

Because (3.1) is satisfied for arbitrary solenoidal ú, $\mathbf{\Phi}$ must have a scalar potential $\varphi$. By introducing a stream function $\psi$ such that $[\grave{u}, \grave{v}]=\left[\psi_{r}+r^{-1} \psi,-\psi_{x}\right]$ and eliminating the potential $\varphi$ by combining the axial and radial equations of $\nabla \varphi=\boldsymbol{\Phi}$, we find that $\psi$ satisfies

$$
\begin{gathered}
\psi_{x x x x}+2 \psi_{x x r r}+2 r^{-1} \psi_{x x r}-2 r^{-2} \psi_{x x} \\
+\psi_{r r r r}+2 r^{-1} \psi_{r r r}-3 r^{-2} \psi_{r r}+3 r^{-3} \psi_{r}-3 r^{-4} \psi= \\
\frac{R}{2 \grave{m}} \partial_{x}\left(-2 \psi_{r} U_{B}^{\prime}-r^{-1} \psi U_{B}^{\prime}-\psi U_{B}^{\prime \prime}\right),
\end{gathered}
$$

together with $\psi=\psi_{r}=0$ at $r=r_{a}$ and $r=r_{b}$. Note that the solution can be taken to be proportional to $e^{\mathrm{i} \alpha x}$ because equation (3.2) is now linear. As a consequence, we can solve this equation numerically by expanding $\psi$ in terms of Chebyshev polynomials of the first kind $T_{l}(y)$ as

$$
\psi(x, y)=\sum_{l=0}^{L} X_{l}\left(1-y^{2}\right)^{2} T_{l}(y) e^{\mathrm{i} \alpha x}
$$

and evaluating (3.2) at the collocation points

$$
y_{l}=\cos \left(\frac{l+1}{L+2} \pi\right), l=0, \ldots, L
$$

Here

$$
y=r-r_{m} \in[-1,1]
$$

is the mapped radial coordinate, with $r_{m}=\frac{1+\eta}{1-\eta}$ representing the mid-gap. The resultant algebraic eigenvalue problem

$$
A_{l j} X_{j}=\sigma B_{l j} X_{j}
$$

leads to $L+1$ eigenvalues $\sigma \equiv \alpha R / \grave{m}$. The minimum value of positive purely real $\sigma$ gives $\alpha R_{E}$ where $R_{E}$ is the energy Reynolds number. There are no finite-amplitude solutions if $R<R_{E}$, because all disturbances monotonically decay.

The results of numerical computations are shown in figure 1 . We investigated the range $0.01 \leq \eta \leq 1$ and found that $\alpha R_{E}$ increases with decreasing $\eta$. Owing to the transformation (3.4) and the specific potential form chosen here, (3.2) reduces to its planar counterpart in the narrow-gap limit $\eta \rightarrow 1$. Because $\sigma(\alpha, \eta)=\sigma(-\alpha, \eta), \alpha R_{E}$ must have a local extremal value at $\alpha=0$. From the figure, we can see that this point is a global minimum for the current range of $\eta$. However, we note that the minimum of $R_{E}$ is at a non-zero value of $\alpha$, e.g. for $\eta=0.1$, the minimum $R_{E}=122$ occurs at $\alpha=1.96$.

The axisymmetric finite-amplitude solutions found by DN11 have $\alpha=O(1)$ and exist over a range of $R=O\left(10^{4}\right)$, comfortably in excess of the energy Reynolds numbers calculated here. For example, the branch of the axisymmetric solution for $\alpha=0.6546$ discovered by DN11 has a turning point at $R=4.0 \times 10^{4}$, which is considerably larger than the corresponding $R_{E}=224$. We revisit the energy analysis in section 7 when considering finite amplitude solutions in the limit $\alpha \rightarrow 0$, where we find that the bound obtained here turns out to be surprisingly sharp in this case. 


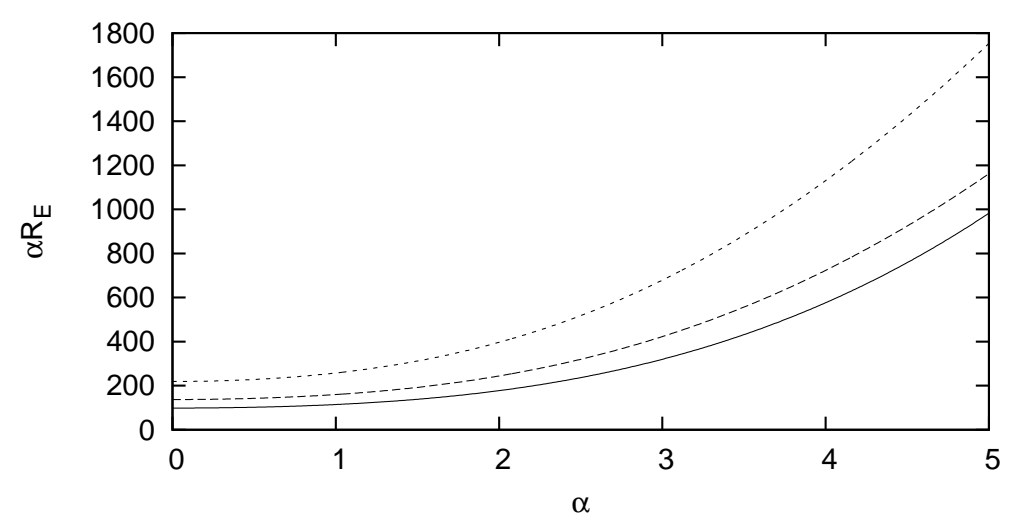

Figure 1. The energy Reynolds number $R_{E}$. The solid, dashed and dotted lines are calculated for $\eta=1,0.1$ and 0.01 respectively.

\section{Computation of finite-amplitude solutions}

In this section we compute solutions of (2.3)-(2.5) that take the form of travelling waves, propagating downstream with phase speed $c$. The velocities and pressure therefore become functions of the radial variable $r$ and the travelling-wave coordinate

$$
\xi=\alpha(x-c t) \in[0,2 \pi] .
$$

We decompose the disturbance velocity field $[u, v](\xi, r)$ into a spatial mean $[\bar{u}, \bar{v}](r)$ and fluctuation $[\tilde{u}, \tilde{v}](\xi, r)$ where the average operator is defined by

$$
\bar{*} \equiv \frac{1}{2 \pi} \int_{0}^{2 \pi} * d \xi .
$$

Consideration of the continuity equation shows that $\bar{v}=0$ for the mean-flow distortion and that there exists a stream function $\psi$ for the fluctuation $\widetilde{\mathbf{u}}$. From substitution into (2.3), (2.4), (2.5), the governing equations for the variables $\psi$ and $\bar{u}$ are

$$
\begin{gathered}
0=-\left(U_{B}+\bar{u}-c\right)\left(\alpha^{2} \psi_{\xi \xi \xi}+\psi_{\xi r r}+r^{-1} \psi_{\xi r}-r^{-2} \psi_{\xi}\right) \\
-\left(U_{B}^{\prime}+\bar{u}^{\prime}\right) r^{-1} \psi_{\xi}+\left(U_{B}^{\prime \prime}+\bar{u}^{\prime \prime}\right) \psi_{\xi} \\
+(\alpha R)^{-1}\left\{\alpha^{4} \psi_{\xi \xi \xi \xi}+\alpha^{2}\left(2 \psi_{\xi \xi r r}+2 r^{-1} \psi_{\xi \xi r}-2 r^{-2} \psi_{\xi \xi}\right)\right. \\
\left.+\psi_{r r r r}+2 r^{-1} \psi_{r r r}-3 r^{-2} \psi_{r r}+3 r^{-3} \psi_{r}-3 r^{-4} \psi\right\} \\
+\psi_{\xi}\left\{4 r^{-3} \psi-2 r^{-2} \psi_{r}+\psi_{r r r}+\alpha^{2}\left(\psi_{\xi \xi r}-r^{-1} \psi_{\xi \xi}\right)\right\} \\
-\left(\psi_{r}+r^{-1} \psi\right)\left(\alpha^{2} \psi_{\xi \xi \xi}+\psi_{\xi r r}+r^{-1} \psi_{\xi r}\right)
\end{gathered}
$$

and

$$
0=r^{-1} \partial_{r}\left\{r \overline{\psi_{\xi}\left(\psi_{r}+r^{-1} \psi\right)}+(\alpha R)^{-1} r \bar{u}^{\prime}\right\}
$$

together with the boundary condition

$$
\psi=\psi_{r}=\bar{u}=0 \text { at } r=r_{a} \text { and } r=r_{b} .
$$

For numerical purposes, $\psi$ and $\bar{u}$ are expanded by using the gap coordinate $y$ defined 
in (3.4). They take the form

$$
\begin{aligned}
\psi(\xi, y) & =\sum_{n=1}^{N} \sum_{l=0}^{L} X_{j(l, n)}\left(1-y^{2}\right)^{2} T_{l}(y) e^{\mathrm{i} n \xi}+\text { c.c. } \\
\bar{u}(y) & =\sum_{l=0}^{L} X_{j(l, 0)}\left(1-y^{2}\right) T_{l}(y)
\end{aligned}
$$

where $j(l, n) \equiv(l+1) \times(n+1)$ and c.c. denotes complex conjugate. The $j$ th equation is found by evaluating the values of $\overline{e^{-\mathrm{i} n \xi}(4.2)}$ for $n=1, \ldots, N$ and (4.3) for $n=0$ at the same collocation points $y_{l}(l=0, \ldots L)$ used in (3.3). The unknown phase velocity $c$ is added as the top entry in the solution vector, and the corresponding additional equation is the spatial phase lock condition which we impose here as

$$
F(0)-\text { c.c. }=0 \text {, }
$$

where the first Fourier coefficient of the axial velocity is defined as

$$
F(y) \equiv 2 \overline{u e^{-\mathrm{i} \xi}}
$$

This ensures that the $\sin \xi$ component of $u$ vanishes at the midpoint of the gap. The problem has now been reduced to a system of quadratic algebraic equations of the form

$$
D_{k j} X_{j}+H_{k j j^{\prime}} X_{j} X_{j^{\prime}}=0 .
$$

Noting that the first $L+2$ unknowns/equations are purely real whereas the remainder are complex, we use a Newton iterative method of dimension $(2 N+1) \times(L+1)+1$ to obtain the solution. Any signal-to-noise-floor ratio of the converged solutions, i.e. $\left\|D_{k j} X_{j}+H_{k j j^{\prime}} X_{j} X_{j^{\prime}}\right\|_{2} /\left\|D_{k j} X_{j}\right\|_{2}$, is less than $10^{-10}$ throughout the paper. In our computational approach, all Jacobian elements are explicitly given by straightforward algebra and are used to update the Newton guess together with an LAPACK direct linear solver routine. The linear problems, which consume most of the computational time, are quite massive, but recent advances in computer power allows us to solve them. Further detail of the method of computation can be found in DN11.

\subsection{Results}

If $\eta \lesssim 0.1415$, where the long-wave cut-off of linear instability occurs (Gittler 1993), the basic flow can be destabilized by axisymmetric infinitesimal disturbances. Because the linear approximation of the growing mode is accurate sufficiently close to the neutral stability point, it can be used as an initial guess for a nonlinear calculation involving Newton's method. As a consequence, the solution branch of the finite-amplitude axisymmetric travelling wave bifurcates from this point. Here we introduce the normalized momentum transport on the wall $M$ to measure how much a finite-amplitude solution differs from the basic state. Note that $\left.r_{a} U_{B}^{\prime}\right|_{r=r_{a}}=\left.r_{b} U_{B}^{\prime}\right|_{r=r_{b}}$ and $\left.r_{a} \bar{u}^{\prime}\right|_{r=r_{a}}=\left.r_{b} \bar{u}^{\prime}\right|_{r=r_{b}}$, by (2.2) and (4.3). This enables us to define $M$ as

$$
\left.M \equiv \frac{\bar{u}^{\prime}+U_{B}^{\prime}}{U_{B}^{\prime}}\right|_{r=r_{a}}=\left.\frac{\bar{u}^{\prime}+U_{B}^{\prime}}{U_{B}^{\prime}}\right|_{r=r_{b}} .
$$

Figure 2 shows the bifurcation diagram for $\eta=0.1$. For this radius ratio, it is known that the linear instability has a critical Reynolds number $R=3.6 \times 10^{6}$, and this occurs for $\alpha=0.6546$. It is also known that the axisymmetric solution subcritically bifurcates from this neutral point (DN11). We discover that at $R=1.6 \times 10^{7}$, the base flow again becomes stable at this value of wavenumber. From this second neutral point, another 
(a)

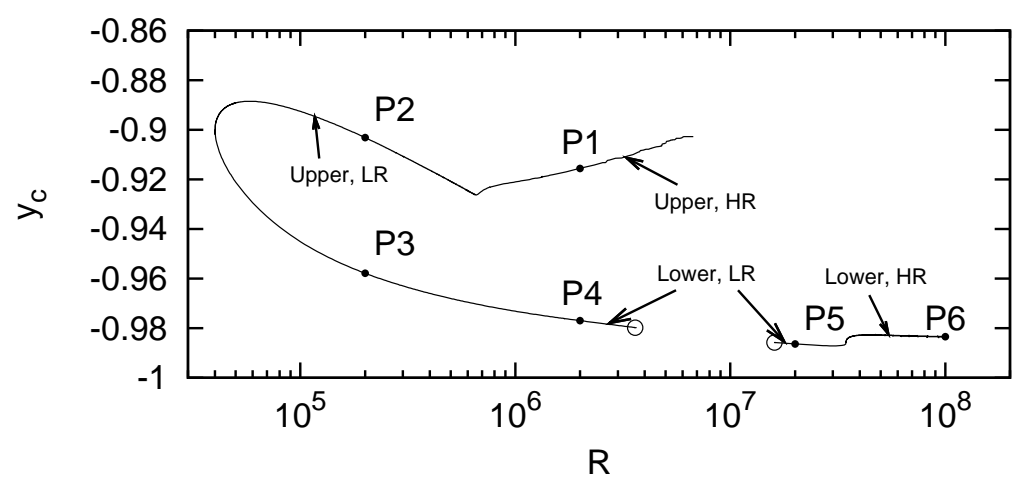

(b)

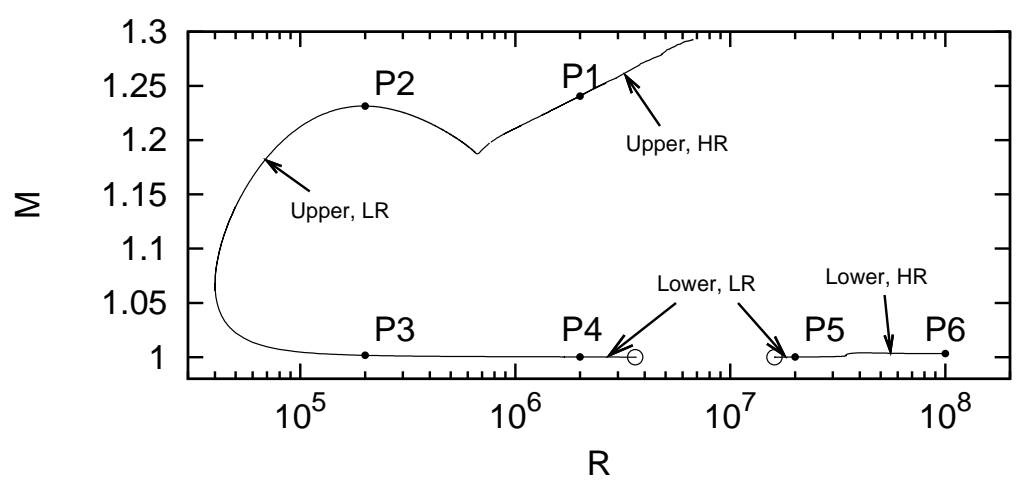

FIGURE 2. The axisymmetric travelling-wave bifurcation diagram for $(\eta, \alpha)=(0.1,0.6546)$. The upper and lower figures represent the variation of the critical layer location $y_{c}$ and the momentum transport $M$ respectively. Open circles in the figures represent linear critical points. The range of truncation level $L \in[120,200]$ and $N \in[30,60]$ is used to compute the solution curves.

bifurcation of the axisymmetric solution is detected. This bifurcation scenario is also pictured in figure 2, and it can be seen in the figure that the resultant branch heads in the direction of increasing $R$ where the basic flow again becomes linearly stable, i.e. the new bifurcation is also subcritical. We adopt the terminology 'lower branch' to represent the branch that passes through the neutral points, and the points P3-P6 while the phrase 'upper branch' is used to denote solutions at higher $M$, that lie on the solution curve that passes through $\mathrm{P} 1$ and $\mathrm{P} 2$. One of the striking features of the lower branch is the kink at $R \simeq 3.5 \times 10^{7}$, and a similar kink can also be found on the upper branch in between the points $\mathrm{P} 2$ and $\mathrm{P} 1$, at $R \simeq 6.7 \times 10^{5}$. The branches behave differently before and after the kinks, although no bifurcations take place here, and the curves appear smooth when examined closely. Henceforth we denote the branches before and after the kinks as the LR (Low Reynolds number) and HR (High Reynolds number) modes respectively.

Along the branches, the streamwise fluctuation flow-field, $\tilde{u}$, is visualized in figures 3 and 4 . The plots labelled $\mathrm{P} 1-\mathrm{P} 6$ represent solutions at the corresponding points in figure 2. For the upper branch solution at sufficiently high Reynolds number (P1, P2), we can see a relatively slowly-varying, evenly-placed strong positive/negative pattern in the vicinity of the critical layer $y_{c}$, where the basic flow speed, $U_{B}$, coincides with the phase velocity $c$ of the travelling-wave solution. In these cases, the critical layer is situated near the inner wall: the distance between the critical layer and inner wall is 
$\mathrm{P} 1: R=2 \times 10^{6}$ upper branch

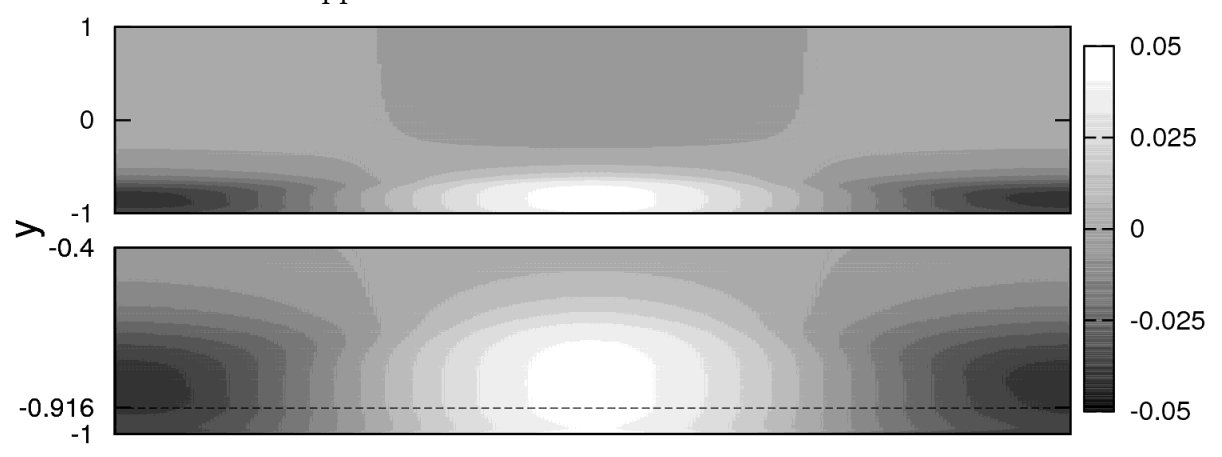

P2: $R=2 \times 10^{5}$ upper branch

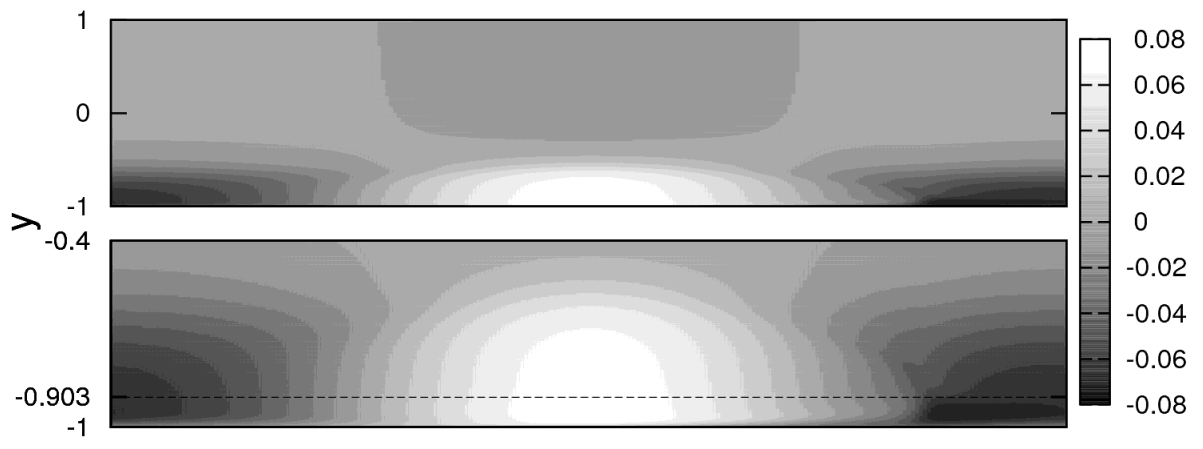

P3: $R=2 \times 10^{5}$ lower branch

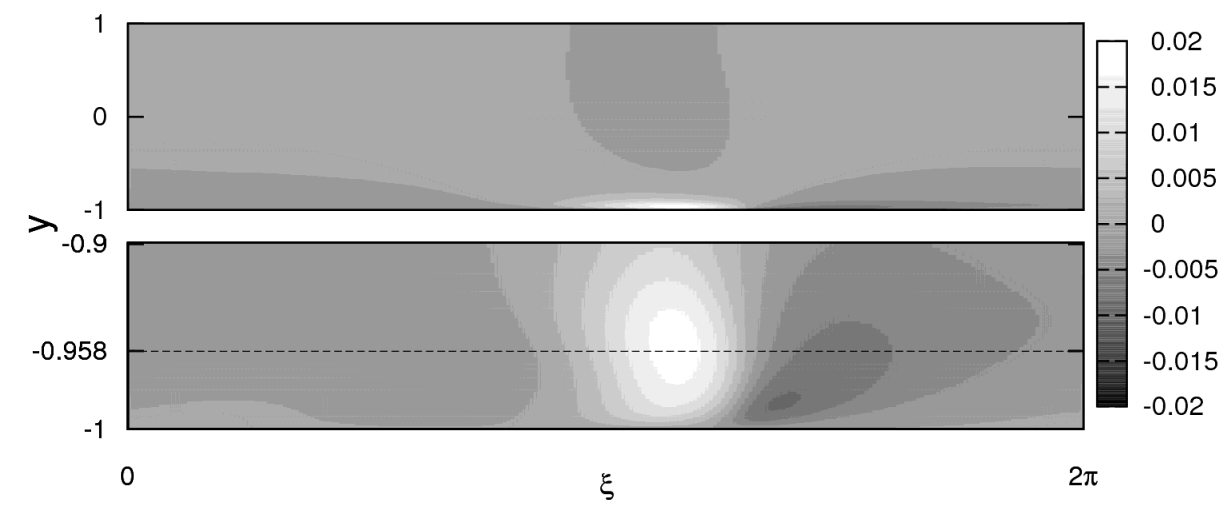

FiguRE 3. The axial fluctuation velocity field $\tilde{u}$ for $(\eta, \alpha)=(0.1,0.6546)$. The dashed lines in the close-up figures, which are placed just below the full domain representation in each case, represent the critical layer locations $y_{c}$. P1, P2 and P3 correspond to the points in figure 2 .

only $5 \%$ of the gap (see figure $2(\mathrm{a})$ ). As we move towards the point P3, which lies on the lower branch, the positive part of the flow pattern begins to be squeezed in the axial direction. Then as $R$ is increased along the lower branch (P4, P5), the critical layer moves even closer to the inner wall (typically it is now at $1 \%$ of the gap from the inner wall) and the strong positive/negative flow pattern is again evenly-placed, although it is now much more concentrated than it was on the upper branch. The magnitude of the solution decreases as the branch approaches the linear instability, but it begins to grow again after the linear neutral point is passed (P6). The computation of the solution 


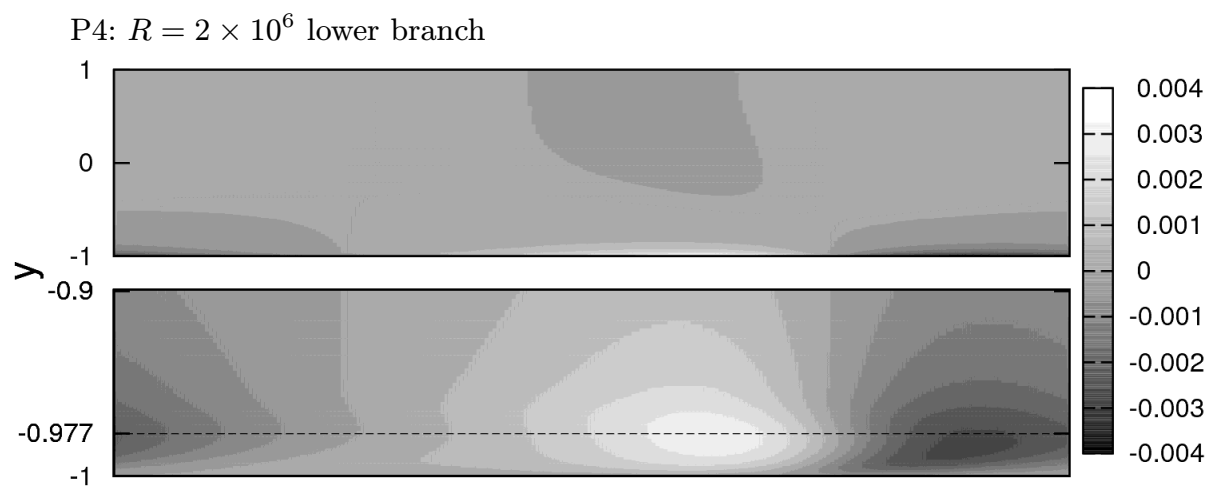

P5: $R=2 \times 10^{7}$ lower branch

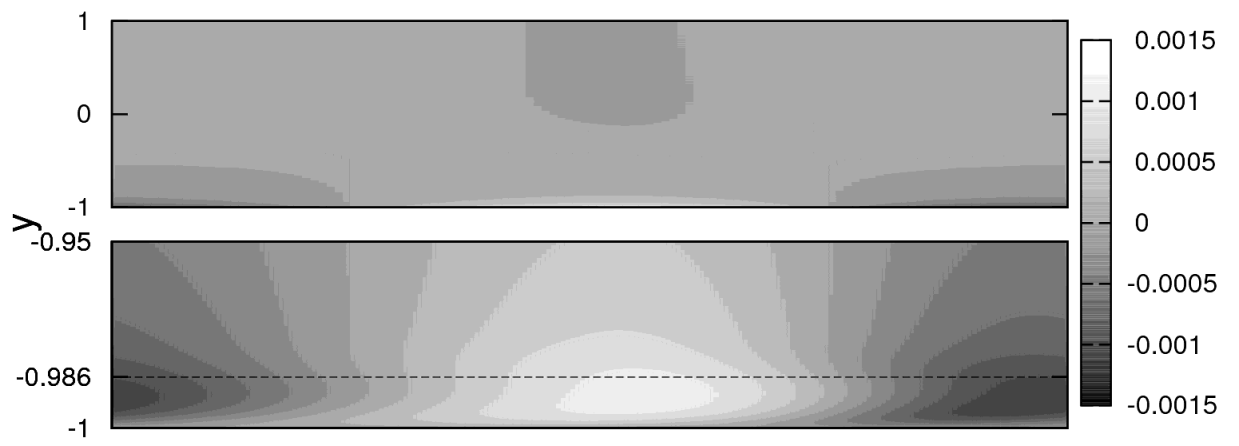

P6: $R=10^{8}$ lower branch

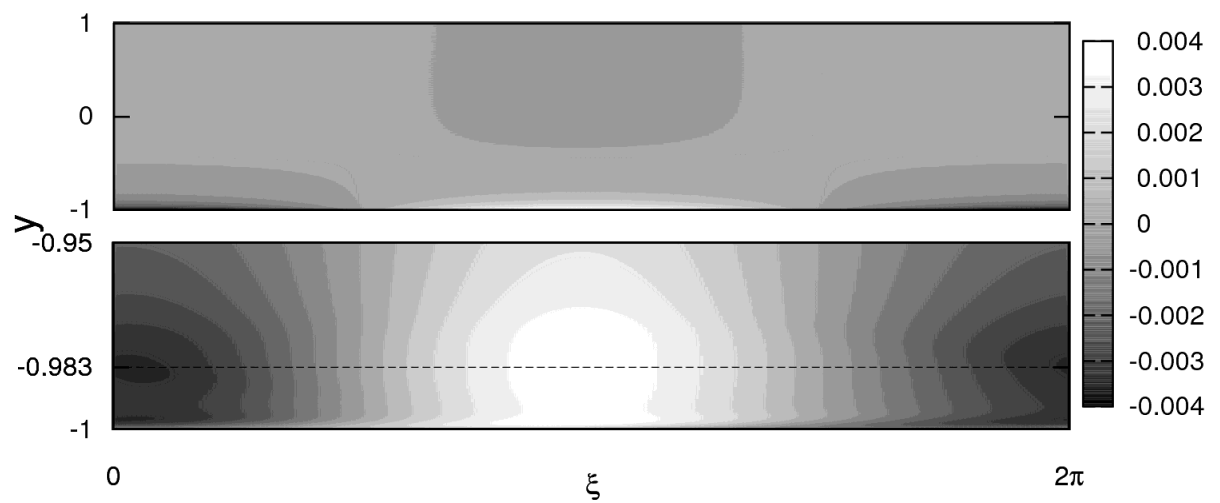

FiguRE 4. Same parameter values as figure 3 but for the solutions at the points P4, P5, P6.

beyond $\mathrm{P} 6$, where $R$ is in excess of $10^{8}$, is very difficult because of the Gibbs phenomena of the Chebyshev basis due to the very sharp structure near to $y_{c}$. This feature can also be found in the mean-flow distortion plot for P1-P6, which is presented in figure 5 . When we examine the high Reynolds number structure of this mode in the next section we will see that a thin critical layer, centred at $y=y_{c}$, regularizes this apparent singularity.

To examine the geometry dependence of the travelling-wave solutions, the solution branches are calculated for various $\eta$ and $R$, fixing $\alpha=0.6546$. The results are shown in figure 6 , where the solution branches, shown as solid lines, bifurcate from the thick dashed curve traced in the $M=1$ plane, which represents the linear neutral curve for $\alpha=0.6546$. When $\eta$ is increased to 0.14 , The HR mode and the LR mode are separated 

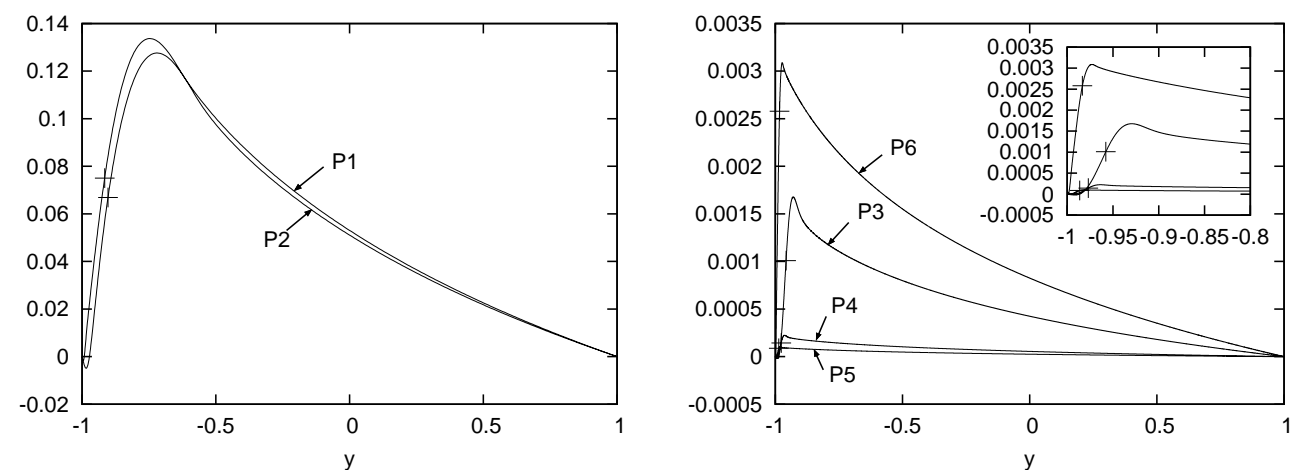

Figure 5. The mean-flow distortion $\bar{u}$. Left and right figures correspond to upper and lower branch solutions. The crosses on the curves represent the critical layer locations $y_{c}$.

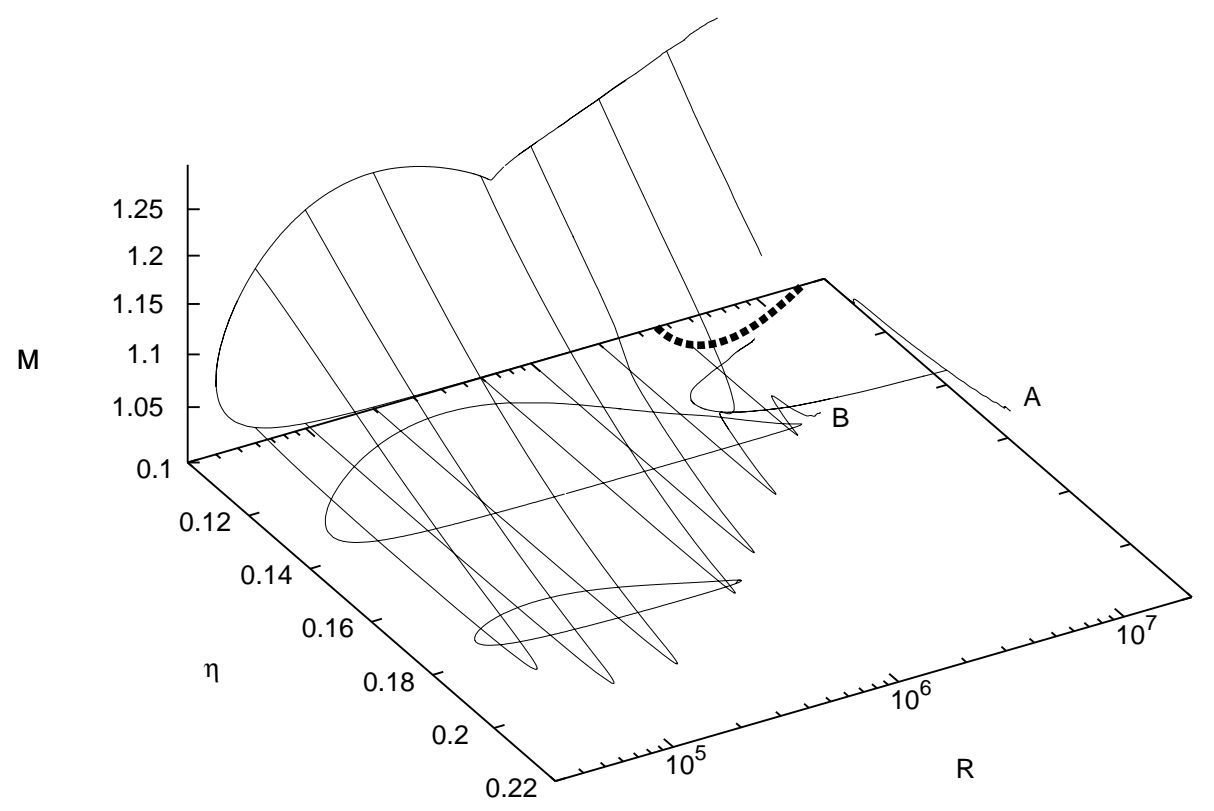

FIgURE 6 . The bifurcating branches of the axisymmetric travelling-wave solutions (solid lines) from the linear neutral curve (thick dashed line) with $\alpha=0.6546$. The base level is placed at $M=1$. The range of truncation level $L \in[120,200]$ and $N \in[30,60]$ is used to compute the solution curves.

into an open branch and a closed branch respectively. Due to this separation, the branch has three turning points when $\eta$ is varied at fixed sufficiently high $R$. Since we originally defined the HR and LR modes in terms of their position with respect to the kink, it seems reasonable to consider the first turning point encountered as $M$ is increased from unity to represent the existence boundary for the LR mode. This boundary defines a critical value of $\eta$, beyond which the LR mode ceases to exist, and its variation with $R$ is plotted in figure 7 for $\alpha=0.6546,0.2,0.1$ and 0.05 . The linear neutral curves and 


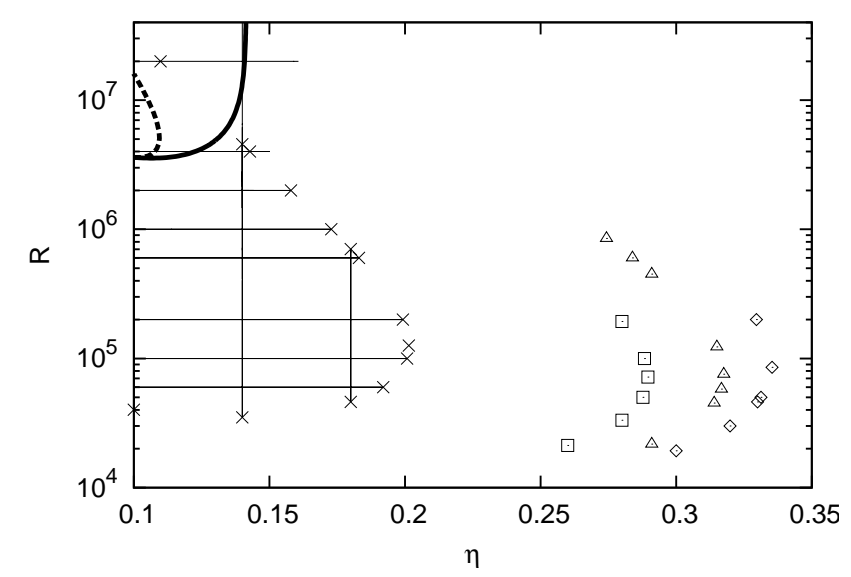

FIGURE 7. First turning points from the lower branch, which represent the edge of the existence of the LR mode. $\times, \square, \triangle$ and $\diamond$ are calculated with $\alpha=0.6546,0.2,0.1$ and 0.05 respectively. The lines in the figure are the projection of the solution curves in figure 6 . The thick solid line represents the linear neutral curve taken over all wavenumbers.

the branches of the solution in figure 6 are also projected on this figure. We can see clearly that the solution can exist well beyond the long-wave cut-off of linear instability, where the thick solid line heads sharply towards $R=\infty$ at $\eta=0.1415$, and the branch extends to larger $\eta$ as $\alpha$ is decreased. The maximum $\eta$ of the LR mode is at relatively low $R$ for all $\alpha$, and then it monotonically decreases for higher $R$. In contrast, the present calculations suggest that the existence region of the HR mode expands as $R$ is increased. We cannot be certain about this however, because there are resolution issues associated with continuing the branches marked 'A' and 'B' beyond that indicated in figure 6 .

\section{Asymptotic solution at large Reynolds number}

In this section we propose a nonlinear equilibrium travelling-wave structure for annular sliding Couette flow, valid for asymptotically large values of the Reynolds number. The structure consists of a core region of $O(1)$ radial extent in which the flow dynamics are linear and inviscid to leading order, with viscous wall layers adjacent to the inner and outer cylinders. We assume that the wavenumber $\alpha$ is $O(1)$, with the unknown real wavespeed $c \sim O(1)$, and $1-c \sim O(1)$ also. As a consequence, a singularity is encountered within the core region at the location $r_{c}$ where $U_{B}\left(r_{c}\right)=c$. This singularity is regularized within a thin nonlinear critical layer. The phase shifts induced across the two wall layers must be balanced by the corresponding phase shift induced across the critical layer, and this requirement leads to the determination of the amplitude of the disturbance in terms of its axial wavenumber and the properties of the basic flow. A sketch of the flow structure is shown in Figure 8. The structure is similar to that found by Smith \& Bodonyi (1982a) for fully-developed flow through a single pipe, and Walton $(2002,2003)$ for impulsivelystarted pipe flow and pressure-driven annular Couette-Poiseuille flow. A key difference to the previous studies is the assumption of axisymmetry. We find that unlike for the single pipe case, axisymmetric solutions are indeed possible here. One of the reasons for this is that, for the fully-developed flow through a circular pipe, the combination $U_{B}^{\prime \prime}-U_{B}^{\prime} / r$ is zero, in contrast to the flow under consideration here, where this quantity remains positive throughout the annulus. It is the non-zero nature of this term that gives rise to the logarithmic singularity referred to above, and evident in (5.9) below. The same 


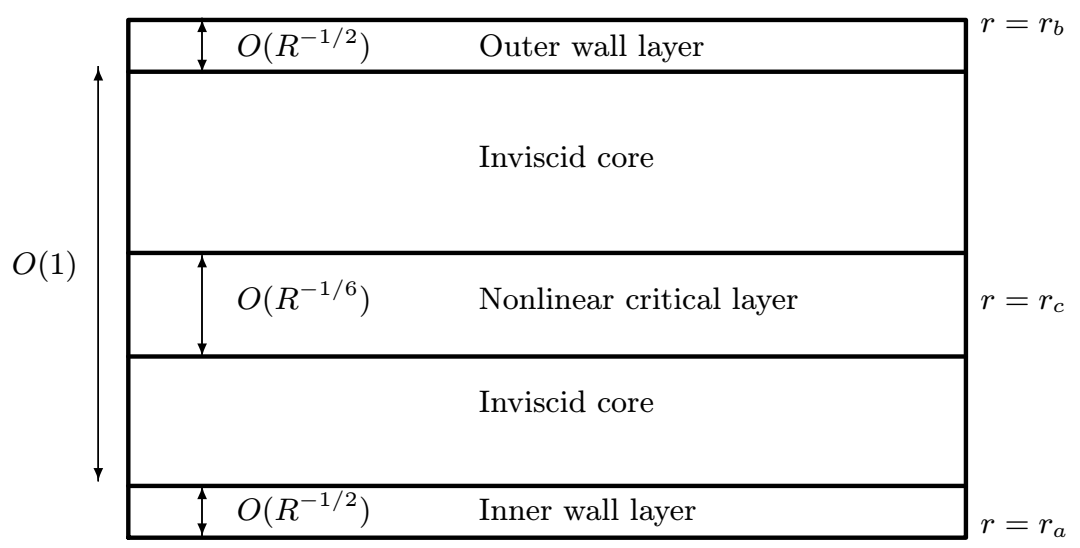

FIGURE 8. Sketch of the high Reynolds number asymptotic travelling-wave structure for annular sliding Couette flow.

arguments mean that this structure is also absent in plane Couette flow, implying that the asymptotic structure presented here does not exist in the narrow gap limit.

The flow behaviour in the various regions is set out in the following sub-sections.

\subsection{The inviscid core flow}

In the core, the flow dynamics are inviscid to leading order, with the flow expansions assuming the form

$$
\begin{aligned}
u= & \varepsilon \bar{u}_{1}(r)+\varepsilon^{2}\left\{A_{0} F_{2}(r) \cos \xi+\bar{u}_{2}(r)\right\} \\
& +\cdots+\varepsilon^{2} R^{-1 / 2} u_{5}(\xi, r)+\cdots, \\
v= & -\varepsilon^{2} A_{0} G_{2}(r) \sin \xi+\cdots+\varepsilon^{2} R^{-1 / 2} v_{5}(\xi, r)+\cdots, \\
p= & \varepsilon^{2} A_{0} P_{2}(r) \cos \xi+\cdots+\varepsilon^{2} R^{-1 / 2} p_{5}(\xi, r)+\cdots .
\end{aligned}
$$

Here $\bar{u}_{1}(r)$ is the leading-order mean-flow distortion term and $\varepsilon$ is a small parameter that will be determined in terms of the Reynolds number subsequently. The real constant $A_{0}$ is also to be determined, and the variable $\xi$ is the same travelling-wave coordinate defined in (4.1). The terms with subscript 5 are the highest-order terms that break the [even, odd, even] symmetry of $[u, v, p]$ about $\xi=\pi$, i.e.

$$
\left[u_{5}, v_{5}, p_{5}\right] \text { contain terms } A_{0}\left[F_{5}(r) \sin \xi, G_{5}(r) \cos \xi, P_{5}(r) \sin \xi\right] .
$$

In order to fix the phase of the solution we impose the phase normalization condition

$$
P_{2}\left(r_{c}\right)=1,
$$

where $r=r_{c}$ is the location of the critical layer, i.e. the radial location where $U_{B}\left(r_{c}\right)=$ $c$, with $U_{B}$ the basic flow defined in (2.2). The choice of (5.3) is mainly for algebraic simplicity. If we do not impose this condition then the quantity $P_{2}\left(r_{c}\right)$ will appear in expressions throughout the critical-layer analysis below.

\subsubsection{The core-flow fluctuation}

Substitution of (5.1) into the Navier-Stokes equations (2.3)-(2.5) leads to the following inviscid balances:

$$
\left(U_{B}(r)-c\right) \alpha F_{i}+G_{i} U_{B}^{\prime}=-\alpha P_{i}, \quad\left(U_{B}(r)-c\right) \alpha G_{i}=P_{i}^{\prime},
$$


for $i \in\{2,5\}$, together with the continuity equation. Further manipulation and elimination of the velocity components leads to Rayleigh equations for the pressure components

$$
\left(U_{B}(r)-c\right)\left(P_{i}^{\prime \prime}+r^{-1} P_{i}^{\prime}-\alpha^{2} P_{i}\right)=2 U_{B}^{\prime} P_{i}^{\prime}, \quad i \in\{2,5\} .
$$

For the $P_{2}$-component, the appropriate boundary conditions are

$$
P_{2}^{\prime}\left(r_{a}\right)=P_{2}^{\prime}\left(r_{b}\right)=0
$$

which arise from imposition of the condition of zero radial velocity at the wall. For the $P_{5}$-component, the wall-layer analysis (section 5.2) predicts the existence of a non-zero radial velocity component of $O\left(\varepsilon^{2} R^{-1 / 2}\right)$ as each wall is approached, implying that the appropriate conditions on the radial fluctuation in the core are

$$
G_{5}\left(r_{a}\right)=g_{a}, G_{5}\left(r_{b}\right)=g_{b}
$$

with the precise values of $g_{a}$ and $g_{b}$ to be fixed in (5.26), (5.32). In terms of the pressure, the boundary conditions are therefore

$$
P_{5}^{\prime}\left(r_{a}\right)=(1-c) \alpha g_{a}, \quad P_{5}^{\prime}\left(r_{b}\right)=-\alpha c g_{b}
$$

from the radial momentum balance in (5.4).

For the purposes of the critical-layer analysis to be presented in section 5.3, we need to know the limiting behaviour of the flow as the critical layer is approached. This can be calculated by the Frobenius method, and the relevant asymptotes, as $r \rightarrow r_{c} \pm$, are:

$$
\begin{aligned}
& P_{i} \sim P_{i}\left(r_{c}\right)\left[1-\frac{\alpha^{2} r_{c}^{2}}{2}\left(\frac{r_{c}-r}{r_{c}}\right)^{2}-\frac{2 \alpha^{2} r_{c}^{2}}{3}\left(\frac{r_{c}-r}{r_{c}}\right)^{3}\left(\ln \left|\frac{r_{c}-r}{r_{c}}\right|+j_{ \pm}^{(i)}\right)+\cdots\right] \\
& F_{i} \sim 2 \tau_{0}^{-1} P_{i}\left(r_{c}\right)\left[\ln \left|\frac{r_{c}-r}{r_{c}}\right|+j_{ \pm}^{(i)}+\frac{1}{6}\left(1+\frac{5 \tau_{1}}{\tau_{0}}\right)+\cdots\right]
\end{aligned}
$$

(with a similar expansion for $G_{i}$ ) for $i \in\{2,5\}$, with the \pm denoting the limits $r \rightarrow r_{c} \pm$. Here we have defined

$$
\tau_{0}=-r_{c} U_{B}^{\prime}\left(r_{c}\right)=-(\ln \eta)^{-1}, \tau_{1}=\left(r_{c}^{2} / 2\right) U_{B}^{\prime \prime}\left(r_{c}\right)=-(2 \ln \eta)^{-1},
$$

to represent the shear and curvature of the basic flow at the critical location, and we have made use of the property $2 \tau_{1} / \tau_{0}=1$ to simplify the expressions slightly. The constants $j_{ \pm}^{(i)}$ are determined by solving the Rayleigh equation for $P_{i}$ numerically. This is described for $P_{2}$ in more detail in section 5.1 .2 below. A feature of the nonlinear critical layer is the smallness of the jump in velocity and pressure induced across it, in contrast with a classical linear critical layer in which the phase shift is $O(1)$. This feature can be anticipated here by taking $j_{+}^{(2)}=j_{-}^{(2)}$. However it will be necessary for $j_{+}^{(5)}-j_{-}^{(5)}$ to be non-zero in order to accommodate the velocity jump across the critical layer. We shall see that the critical layer analysis shows that there is a jump

$$
\varepsilon^{2} R^{-1 / 2} \phi \sin \xi
$$

in the streamwise velocity as we cross the critical layer from $r=r_{c}-$ to $r=r_{c}+$, with $\phi$ determined specifically in (5.52). In terms of the core properties, we therefore have

$$
\phi=2 A_{0} P_{5}\left(r_{c}\right) \tau_{0}^{-1}\left(j_{+}^{(5)}-j_{-}^{(5)}\right)
$$

in view of the asymptotic expansion for $F_{5}$ in $(5.9)$. The quantity $\left(j_{+}^{(5)}-j_{-}^{(5)}\right)$ can be related to the wall velocities $g_{a}, g_{b}$, by considering the Wronskian of the solutions to (5.5), 
which has the form

$$
r\left(U_{B}(r)-c\right)^{-2}\left(P_{5} P_{2}^{\prime}-P_{2} P_{5}^{\prime}\right)=\omega_{ \pm}
$$

Applying the boundary conditions (5.6), (5.8), we can determine the constants $\omega_{ \pm}$as

$$
\omega_{+}=\alpha g_{b} r_{b} P_{2}\left(r_{b}\right) / c, \omega_{-}=-\alpha g_{a} r_{a} P_{2}\left(r_{a}\right) /(1-c) .
$$

Then, letting $r \rightarrow r_{c} \pm$ in (5.13), using the series expansions in (5.9) for $P_{2}$ and $P_{5}$, and the phase normalization (5.3) we find that (5.12) can be rewritten as

$$
\phi=-\left(\omega_{+}-\omega_{-}\right) \alpha^{-2} r_{c}^{-2} A_{0} \tau_{0}
$$

so that the velocity jump across the critical layer is now related to the wall-layer properties by (5.14) and (5.15).

\subsubsection{The numerical solution of the Rayleigh equation}

Aside from a number of numerical integrations, the only part of the asymptotic analysis where a non-trivial numerical approach is required is in the solution of the Rayleigh equation (5.5) for $P_{2}(r)$. This solution is important as it yields, for a given wavenumber $\alpha$, the corresponding wavespeed $c$, and hence the location $r_{c}$ of the critical layer. We briefly describe the method we used here for $\alpha$ of $O(1)$ and also the corresponding approach for the limiting case $\alpha \rightarrow 0$.

(i) Solution for $\alpha$ of $O(1)$

First we introduce a new radial variable $s$ such that $r=r_{c} s$ and define $s_{a}=r_{a} / r_{c}, s_{b}=$ $r_{b} / r_{c}, \widehat{\alpha}=\alpha r_{c}$. The Rayleigh equation (5.5) for $P_{2}(r)=Q(s)$ say, can then be rewritten as

$$
Q^{\prime \prime}(s)+\frac{1}{s} Q^{\prime}(s)-\widehat{\alpha}^{2} Q(s)=\frac{2}{s}(\ln s)^{-1} Q^{\prime}(s), Q^{\prime}\left(s_{a}\right)=Q^{\prime}\left(s_{b}\right)=0, Q(1)=1 .
$$

For fixed values of $s_{a}$ and $\widehat{\alpha},(5.16)$ is marched forward from $s=s_{a}$ with a guess for $Q\left(s_{a}\right)$ (equal to $Q_{g}$, say). The values of $Q$ and $Q^{\prime}$ are found at $s=s_{1}=\left(1+s_{a}\right) / 2$. Equation (5.16) is then marched backwards from $s=s_{2}=1-\varepsilon_{1}$ (with $\varepsilon_{1}$ suitably small) using the series expansion (5.9) to provide values for $Q\left(s_{2}\right), Q^{\prime}\left(s_{2}\right)$. A guess is made for the constant $j_{-}^{(2)}$. As a result of this procedure we can calculate a second set of values for $Q\left(s_{1}\right), Q^{\prime}\left(s_{1}\right)$. We then iterate on the guesses $Q_{g}$ and $j_{-}^{(2)}$ until the two estimates for $Q$ and $Q^{\prime}$ at $s_{1}$ are in agreement. Next we set $j_{+}^{(2)}=j_{-}^{(2)}$ (as discussed just below (5.10)) and march (5.16) forward from $s=1+\varepsilon_{1}$, again using the series expansion for $P_{2}$ in $(5.9)$ to provide suitable starting values. The marching process is stopped when the location $s_{b}$ is reached at which $Q^{\prime}\left(s_{b}\right)=0$. The required value for the critical layer location is then given by $r_{c}=r_{b} s_{b}^{-1}$. The wavespeed follows from $c=U_{B}\left(r_{c}\right)$ and the corresponding wavenumber and radius ratio from $\alpha=r_{c}^{-1} \widehat{\alpha}, \eta=s_{a} / s_{b}$. The pressure at both walls is also required later and so these values are also stored. Clearly this procedure can be repeated for a range of wavenumbers and radius ratios.

(ii) Solution in the limit $\alpha \rightarrow 0$

In addition to providing a partial check on the accuracy of the numerical procedure outlined in part (i), the form of the solution of the Rayleigh equation (5.5) as $\alpha \rightarrow 0$ is significant as it provides an important clue as to the form of the new long-wave solution structure that emerges in place of the present structure when $\alpha \sim O\left(R^{-1}\right)$. This structure is discussed in some detail in section 5.4 and draws on the asymptotic forms set out here.

If we consider the inviscid disturbance equations (5.4) in the core, together with the 
continuity balance, we find that as $\alpha \rightarrow 0$ :

$$
F_{2} \sim F_{20}(r)+O\left(\alpha^{2}\right), G_{2} \sim \alpha G_{20}(r)+O\left(\alpha^{3}\right), P_{2} \sim P_{20}+O\left(\alpha^{2}\right),
$$

where $P_{20}$ is a constant. The leading-order terms $\left(F_{20}, G_{20}, P_{20}\right)$ satisfy the balances

$$
F_{20}+G_{20}^{\prime}+G_{20} / r=0,\left(U_{B}(r)-c\right) F_{20}+G_{20} U_{B}^{\prime}=-P_{20},
$$

and elimination of $F_{20}$ leads to the result

$$
\frac{r G_{20}}{\left(U_{B}-c\right)}= \begin{cases}P_{20} \int_{r_{a}}^{r} s\left(U_{B}(s)-c\right)^{-2} d s, & \left(r<r_{c}\right), \\ P_{20} \int_{r_{b}}^{r} s\left(U_{B}(s)-c\right)^{-2} d s, & \left(r>r_{c}\right) .\end{cases}
$$

Across the critical layer we have a zero jump in the component $G_{20}$ and this implies that the following integral condition must hold:

$$
f_{r_{a}}^{r_{b}} \frac{r d r}{\left(U_{B}(r)-c\right)^{2}}=0
$$

where the bar denotes the finite part of the integral. Equation (5.18) can be viewed as the $\alpha \rightarrow 0$ limit of the Rayleigh equation (5.5) and determines the solutions for the leading-order wavespeed $c$ for a given value of radius ratio $\eta$. To evaluate the integral numerically we make the substitution $s=r / r_{c}$ as in part (i) and use the specific form for $U_{B}$ given in (2.2). The integral condition (5.18) can then be rewritten as

$$
f_{\eta s_{c}}^{s_{c}} \frac{s d s}{(\ln s)^{2}}=0
$$

which determines the values of the quantity $s_{c}=r_{b} / r_{c}$. The finite-part integral in (5.19) can then be easily evaluated by using integration by parts as, for example, in Appendix B of Walton (2011).

\subsubsection{The mean-flow distortion in the core}

Again, from substitution of the expansions (5.1) into the Navier-Stokes equations $(2.3)-(2.5)$, we find that the leading-order contribution to the mean-flow distortion satisfies

$$
\bar{u}_{1}^{\prime \prime}+r^{-1} \bar{u}_{1}^{\prime}=A_{0}^{2}\left(G_{5} F_{2}^{\prime}-G_{2} F_{5}^{\prime}\right) / 2=0,
$$

using (5.4) and (5.13), so that the distortion is unforced in the core at this order. The solution satisfying no-slip at the walls therefore has the simple form

$$
\bar{u}_{1}= \begin{cases}M_{a} \ln \left(r / r_{a}\right), & \left(r_{a}<r<r_{c}\right), \\ M_{b} \ln \left(r / r_{b}\right), & \left(r_{c}<r<r_{b}\right) .\end{cases}
$$

The solution in the critical layer requires that $\bar{u}_{1}$ be continuous across $r=r_{c}$, but that there is a jump in $\bar{u}_{1}^{\prime}$. We therefore have

$$
M_{a} \ln \left(r_{c} / r_{a}\right)=M_{b} \ln \left(r_{c} / r_{b}\right),
$$

with $M_{b}-M_{a}$ non-zero and to be determined later, in terms of the disturbance amplitude, by the critical-layer analysis. Although the mean flow distortion is unforced in the core, it $i s$ forced in the wall layers, and the specific forms that it takes will be determined in the next sub-section where we consider those layers in some detail. 


\subsection{The viscous wall layers}

Since the dynamics in the core are inviscid to leading order, the leading-order axial fluctuation $F_{2}(r)$ does not tend to zero as $r \rightarrow r_{a}, r_{b}$. Viscous wall layers are therefore required in order that the no-slip condition can be satisfied.

\subsubsection{The boundary-layer on the inner cylinder}

The boundary-layer has the classical $O\left(R^{-1 / 2}\right)$ thickness, and the relevant flow expansions are

$$
\begin{gathered}
U_{B}+u=1+\varepsilon^{2} u_{a}\left(\xi, Z_{a}\right)+R^{-1 / 2} U_{B}^{\prime}\left(r_{a}\right) Z_{a}+\varepsilon^{4} \overline{\mathcal{U}}_{a}\left(Z_{a}\right)+\cdots \\
v=\varepsilon^{2} R^{-1 / 2} v_{a}\left(\xi, Z_{a}\right)+\cdots, p=\varepsilon^{2} A_{0} P_{2}\left(r_{a}\right) \cos \xi+\cdots
\end{gathered}
$$

with $r=r_{a}+R^{-1 / 2} Z_{a}$, where we have anticipated the independence of the pressure on the normal coordinate. The continuity and axial momentum balances for the fluctuation terms are

$$
\alpha \frac{\partial u_{a}}{\partial \xi}+\frac{\partial v_{a}}{\partial Z_{a}}=0, \alpha(1-c) \frac{\partial u_{a}}{\partial \xi}=\alpha A_{0} P_{2}\left(r_{a}\right) \sin \xi+\frac{\partial^{2} u_{a}}{\partial Z_{a}^{2}},
$$

with $u_{a}=v_{a}=0$ on $Z_{a}=0$ and the condition of no exponential growth as $Z_{a} \rightarrow \infty$. The appropriate solutions are

$$
u_{a}=\frac{1}{2} \mathcal{F}_{a}\left(Z_{a}\right) e^{\mathrm{i} \xi}+\text { c.c., } \quad v_{a}=\frac{1}{2} \mathcal{G}_{a}\left(Z_{a}\right) e^{\mathrm{i} \xi}+\text { c.c. }
$$

with

$$
\begin{aligned}
\mathcal{F}_{a}\left(Z_{a}\right) & =-\frac{A_{0} P_{2}\left(r_{a}\right)\left(1-e^{-\mu_{a} Z_{a}}\right)}{(1-c)} \\
\mathcal{G}_{a}\left(Z_{a}\right) & =\frac{\mathrm{i} \alpha A_{0} P_{2}\left(r_{a}\right)\left(Z_{a}+\mu_{a}^{-1}\left(e^{-\mu_{a} Z_{a}}-1\right)\right)}{(1-c)}
\end{aligned}
$$

and $\mu_{a}=(\mathrm{i} \alpha(1-c))^{1 / 2}$. Taking the limit of $(5.25)$ as $Z_{a} \rightarrow \infty$ and matching to the core, we conclude that

$$
g_{a}=-\alpha^{1 / 2} 2^{-1 / 2}(1-c)^{-3 / 2} P_{2}\left(r_{a}\right) .
$$

The mean-flow distortion is forced by the fluctuation and satisfies

$$
\overline{\mathcal{U}}_{a}^{\prime \prime}=\frac{1}{4} \mathcal{G}_{a}^{*}\left(Z_{a}\right) \mathcal{F}_{a}^{\prime}\left(Z_{a}\right)+\text { c.c. },
$$

with $\overline{\mathcal{U}}_{a} \sim M_{a} Z_{a} / r_{a}$ as $Z_{a} \rightarrow \infty$ to match to the core flow (5.20) and $\overline{\mathcal{U}}_{a}(0)=0$ to satisfy the no-slip condition. Use of (5.24), (5.25) for $\mathcal{F}_{a}, \mathcal{G}_{a}$ and integration leads to the explicit expression

$$
\begin{aligned}
\overline{\mathcal{U}}_{a}= & \frac{\left(A_{0} P_{2}\left(r_{a}\right)\right)^{2}}{4(1-c)^{3}}\left(2\left(m_{a} Z_{a}+2\right) \cos \left(m_{a} Z_{a}\right) e^{-m_{a} Z_{a}}\right. \\
& \left.+2\left(m_{a} Z_{a}-1\right) \sin \left(m_{a} Z_{a}\right) e^{-m_{a} Z_{a}}-e^{-2 m_{a} Z_{a}}-3\right)+\frac{M_{a} Z_{a}}{r_{a}},
\end{aligned}
$$

with $m_{a}=\operatorname{Re}\left(\mu_{a}\right)=(\alpha(1-c) / 2)^{1 / 2}$. 


\subsubsection{The boundary-layer on the outer cylinder}

Since the basic flow is zero on the outer cylinder, the appropriate expansion in the upper layer is

$$
\begin{aligned}
& U_{B}+u=\varepsilon^{2} u_{b}\left(\xi, Z_{b}\right)-R^{-1 / 2} U_{B}^{\prime}\left(r_{b}\right) Z_{b}+\varepsilon^{4} \overline{\mathcal{U}}_{b}\left(Z_{b}\right)+\cdots, \\
& v=-\varepsilon^{2} R^{-1 / 2} v_{b}\left(\xi, Z_{b}\right)+\cdots, p=\varepsilon^{2} A_{0} P_{2}\left(r_{b}\right) \cos \xi+\cdots,
\end{aligned}
$$

with $r=r_{b}-R^{-1 / 2} Z_{b}$. The governing equations for the fluctuations and the meanflow distortion are very similar to those in the inner boundary layer and the method of solution proceeds in an identical fashion to yield

$$
u_{b}=\frac{1}{2} \mathcal{F}_{b}\left(Z_{b}\right) e^{\mathrm{i} \xi}+\text { c.c., } \quad v_{b}=\frac{1}{2} \mathcal{G}_{b}\left(Z_{b}\right) e^{\mathrm{i} \xi}+\text { c.c. },
$$

with

$$
\begin{aligned}
\mathcal{F}_{b}\left(Z_{b}\right) & =\frac{A_{0} P_{2}\left(r_{b}\right)\left(1-e^{-\mu_{b} Z_{b}}\right)}{c}, \\
\mathcal{G}_{b}\left(Z_{b}\right) & =-\frac{\mathrm{i} \alpha A_{0} P_{2}\left(r_{b}\right)\left(Z_{b}+\mu_{b}^{-1}\left(e^{-\mu_{b} Z_{b}}-1\right)\right)}{c},
\end{aligned}
$$

and $\mu_{b}=(-\mathrm{i} \alpha c)^{1 / 2}$. From the expression for $\mathcal{G}_{b}$ we can calculate that

$$
g_{b}=\alpha^{1 / 2} 2^{-1 / 2} c^{-3 / 2} P_{2}\left(r_{b}\right) .
$$

The corresponding solution for the mean-flow distortion is found to be

$$
\begin{aligned}
\overline{\mathcal{U}}_{b}= & -\frac{\left(A_{0} P_{2}\left(r_{b}\right)\right)^{2}}{4 c^{3}}\left(2\left(m_{b} Z_{b}+2\right) \cos \left(m_{b} Z_{b}\right) e^{-m_{b} Z_{b}}\right. \\
& \left.+2\left(m_{b} Z_{b}-1\right) \sin \left(m_{b} Z_{b}\right) e^{-m_{b} Z_{b}}-e^{-2 m_{b} Z_{b}}-3\right)-\frac{M_{b} Z_{b}}{r_{b}},
\end{aligned}
$$

with $m_{b}=\operatorname{Re}\left(\mu_{b}\right)=(\alpha c / 2)^{1 / 2}$. Here we have applied no slip on $Z_{b}=0$ and the corematching condition $\overline{\mathcal{U}}_{b} \sim-M_{b} Z_{b} / r_{b}$ as $Z_{b} \rightarrow \infty$.

Now that we have determined the radial velocities $g_{a}$ and $g_{b}$, we can rewrite the jump condition (5.15) as

$$
\phi=\frac{A_{0} \tau_{0}}{(2 \alpha)^{1 / 2} r_{c}^{2}}\left\{\frac{r_{a}\left[P_{2}\left(r_{a}\right)\right]^{2}}{(1-c)^{5 / 2}}-\frac{r_{b}\left[P_{2}\left(r_{b}\right)\right]^{2}}{c^{5 / 2}}\right\} .
$$

The aim now is to investigate the dynamics of the critical layer with a view to obtaining an alternative, amplitude-dependent expression for $\phi$, and hence determining the amplitude dependence of the neutral modes.

\subsection{The critical-layer analysis}

The aims of the critical-layer analysis are three-fold. The first is to determine the small parameter $\varepsilon$ in terms of the Reynolds number $R$. Next, the jump in the mean-flow distortion across the critical-layer is calculated, and allows us to determine the constants $M_{a}, M_{b}$ introduced in (5.20), thus fixing the core mean-flow distortion. Finally, we find the velocity jump across the layer in terms of the disturbance amplitude $A_{0}$, which can then be determined explicitly as a result. The velocity and pressure expansions are as 
follows

$$
\begin{gathered}
U_{B}+u=c+\varepsilon \widehat{U}_{1}+\left(\varepsilon^{2} \ln \varepsilon\right) \widehat{U}_{2 L}+\varepsilon^{2} \widehat{U}_{2}+\cdots+\varepsilon^{5} \widehat{U}_{5}+\cdots, \\
v=\varepsilon^{2} \widehat{V}_{1}+\left(\varepsilon^{3} \ln \varepsilon\right) \widehat{V}_{2 L}+\varepsilon^{3} \widehat{V}_{2}+\cdots+\varepsilon^{6} \widehat{V}_{5}+\cdots, \\
p=\varepsilon^{2} \widehat{P}_{1}+\varepsilon^{3} \widehat{P}_{2}+\cdots+\varepsilon^{6} \widehat{P}_{5}+\cdots
\end{gathered}
$$

with $r=r_{c}+\varepsilon Y$. The solutions at the first two orders, which match appropriately to the core via (5.1) and (5.9), are relatively simple and can be shown to be

$$
\begin{gathered}
\widehat{U}_{1}=-\tau_{0} Y / r_{c}+\bar{u}_{1}\left(r_{c}\right), \widehat{V}_{1}=-\mu \sin \xi, \widehat{P}_{1}=A_{0} \cos \xi \\
\widehat{U}_{2 L}=2 A_{0} \tau_{0}^{-1} \cos \xi, \widehat{V}_{2 L}=2 \alpha A_{0} \tau_{0}^{-1} Y \sin \xi
\end{gathered}
$$

where the amplitude parameter $\mu$ is defined by

$$
\mu=\alpha r_{c} A_{0} \tau_{0}^{-1} \text {. }
$$

The terms with subscript 2 in (5.35) are the first to possess a non-trivial dependence on the radial coordinate. The governing equations for these terms are

$$
\begin{gathered}
\alpha \widehat{U}_{2 \xi}+\widehat{V}_{2 Y}+\widehat{V}_{1} / r_{c}=0, \widehat{P}_{2 Y}=0, \\
\alpha\left\{\widehat{U}_{1} \widehat{U}_{2 \xi}+\widehat{U}_{2} \widehat{U}_{1 \xi}\right\}+\widehat{V}_{2} \widehat{U}_{1 Y}+\widehat{V}_{1} \widehat{U}_{2 Y}=-\alpha \widehat{P}_{2 \xi},
\end{gathered}
$$

and the match to the core requires

$$
\begin{aligned}
\widehat{U}_{2} \sim & \tau_{1} Y^{2} / r_{c}^{2}+\bar{u}_{1}^{\prime}\left(r_{c} \pm\right) Y+2 A_{0} \tau_{0}^{-1} \ln \left|r_{c}^{-1} Y\right| \cos \xi \\
& +A_{0} \tau_{0}^{-1}\left(2 j^{(2)}+(1 / 3)\left(1+5 \tau_{1} / \tau_{0}\right)\right) \cos \xi
\end{aligned}
$$

as $Y \rightarrow \pm \infty$, in view of (5.9). Differentiating the third of equations (5.39) with respect to $Y$, using (5.36) for $\widehat{U}_{1}, \widehat{V}_{1}$, and switching to a characteristic variable

$$
\zeta=\frac{\alpha \tau_{0}}{2 r_{c}}\left(Y-r_{c} \bar{u}_{1}\left(r_{c}\right) \tau_{0}^{-1}\right)^{2}+\mu \cos \xi,
$$

we eventually obtain the following expression for the shear term $\widehat{U}_{2 Y}$ :

$$
\widehat{U}_{2 Y}=\mp\left(2 \tau_{0} / \alpha r_{c}^{3}\right)^{1 / 2}(\zeta-\mu \cos \xi)^{1 / 2}+\kappa(\zeta) .
$$

An asymptotic condition on the unknown function $\kappa$ can be found by applying the matching condition (5.40), and this yields:

$$
\kappa(\zeta) \sim \pm 2^{3 / 2} \tau_{0}^{1 / 2}\left(\alpha r_{c}^{3}\right)^{-1 / 2} \zeta^{1 / 2}+\Lambda_{ \pm}, \text {as } \zeta \rightarrow \infty
$$

where the constants $\Lambda_{ \pm}$are undetermined at this order. Here, the \pm signs refer to the upper/lower parts of the critical layer wherein $Y-r_{c} \bar{u}_{1}\left(r_{c}\right) \tau_{0}^{-1}>\left(2 r_{c} \mu(1-\cos \xi) / \alpha \tau_{0}\right)^{1 / 2}$, and $Y-r_{c} \bar{u}_{1}\left(r_{c}\right) \tau_{0}^{-1}<-\left(2 r_{c} \mu(1-\cos \xi) / \alpha \tau_{0}\right)^{1 / 2}$, respectively. Later in the analysis it will become clear that $\Lambda_{+} \neq \Lambda_{-}$, and this jump fixes the leading-order mean-flow distortion in the core, via the matching condition (5.40).

The [even, odd, even] symmetry about $\xi=\pi$ of the solution $\left[\widehat{U}_{m}, \widehat{V}_{m}, \widehat{P}_{m}\right]$ is not broken until viscous effects enter the critical layer equations. Since (from (5.36)) we have $\widehat{U}_{1 Y Y}=0$, the first non-zero term of this type is $\widehat{U}_{2 Y Y}$, which makes a contribution at $O\left(R^{-1}\right)$ in the axial momentum equation that determines the quantity $\widehat{U}_{5}$. Balancing inertia and viscosity at this order requires $\varepsilon^{3} R^{-1 / 2} \sim R^{-1}$, and hence fixes the small parameter

$$
\varepsilon=R^{-1 / 6}
$$


If we then carry on to consider the equations at the $m=5$ level and impose periodicity in $\xi$ (which is now a non-trivial condition due to the presence of viscosity and inertia) we can determine fully the unknown function $\kappa$ introduced in (5.42), and we find that

$$
\kappa(\zeta)-\kappa_{0}=\left\{\begin{array}{cc} 
\pm 2^{3 / 2} \pi \tau_{0}^{1 / 2}\left(\alpha r_{c}^{3}\right)^{-1 / 2} \int_{\mu}^{\zeta}\left(I\left(\zeta_{1}\right)\right)^{-1} d \zeta_{1}, & (\zeta>\mu) \\
0, & (\zeta<\mu)
\end{array}\right.
$$

with

$$
I(\zeta)=\int_{0}^{2 \pi}(\zeta-\mu \cos \xi)^{1 / 2} d \xi
$$

The specific form given for $\kappa$ in (5.45) can be deduced from equation (A 1) in Appendix A, together with the asymptotic condition (5.43). A routine numerical calculation then shows that

$$
\Lambda_{+}-\Lambda_{-}=-\left(2 \mu \tau_{0} / \alpha r_{c}^{3}\right)^{1 / 2} C_{0}, \quad C_{0} \simeq 3.90,
$$

and this expression is equal to the distortion shear jump $\bar{u}_{1}^{\prime}\left(r_{c}+\right)-\bar{u}_{1}^{\prime}\left(r_{c}-\right)$, in view of (5.40). This enables us to fix the constants in the core mean flow distortion as

$$
M_{a}=C_{1} \ln \left(r_{c} / r_{b}\right) A_{0}{ }^{1 / 2} / \ln \eta, \quad C_{1}=2^{1 / 2} C_{0} \simeq 5.52,
$$

with $M_{b}$ following from (5.21). The same matching condition also allows us to determine the constant of integration $\kappa_{0}$ in $(5.45)$ as

$$
\kappa_{0}=\left(2 r_{c}\right)^{-1}\left(M_{a}+M_{b}\right)+2 r_{c}^{-1} M_{a} \ln \left(r_{c} / r_{a}\right) .
$$

Since $\kappa$ is now fully determined, it is possible to go back and integrate (5.42) to obtain the explicit expression

$$
\widehat{U}_{2}=-\frac{\zeta}{\alpha r_{c}}+\left(Y-\frac{r_{c} \bar{u}_{1}\left(r_{c}\right)}{\tau_{0}}\right) \kappa(\zeta) \mp\left(\frac{2 r_{c}}{\alpha \tau_{0}}\right)^{1 / 2} \int_{\mu}^{\zeta}(\zeta-\mu \cos \xi)^{1 / 2} \kappa^{\prime}(\zeta) d \zeta+q(\xi),
$$

where

$$
\begin{array}{r}
q(\xi)=\frac{\mu \cos \xi}{\alpha r_{c}}\left\{1+\frac{1}{3}\left(1+\frac{5 \tau_{1}}{\tau_{0}}\right)-2 \ln \left(\alpha \tau_{0} / 2 r_{c}^{3} \mu\right)^{1 / 2}+2 j^{(2)}\right\}+q_{0} \\
+2\left(\alpha r_{c}\right)^{-1} \int_{\mu}^{\infty}\left\{2 \pi(\zeta-\mu \cos \xi)^{1 / 2}(I(\zeta))^{-1}+(\mu \cos \xi)(2 \zeta)^{-1}-1\right\} d \zeta
\end{array}
$$

and $q_{0}$ is a constant, the determination of which would require higher-order analysis. Finally, in order to determine the amplitude $A_{0}$ we need to calculate the phase shift across the critical layer (i.e. the jump in the $\sin \xi$ component of axial velocity across the layer). The calculation is very similar to that performed in Walton (2003), but for the axisymmetric case. The details are given in an appendix, and lead to the expression

$$
\phi=\frac{2 \tau_{0} C_{1}}{\alpha r_{c}^{2} A_{0}^{1 / 2}} .
$$

Equating this to the expression (5.34) for $\phi$ found from the core and wall-layer analyses, enables us to derive an explicit expression for the amplitude dependence of the neutral modes:

$$
A_{0}=\frac{2 \alpha^{-1 / 3} C_{1}^{2 / 3}}{\left(r_{a}\left[P_{2}\left(r_{a}\right)\right]^{2}(1-c)^{-5 / 2}-r_{b}\left[P_{2}\left(r_{b}\right)\right]^{2} c^{-5 / 2}\right)^{2 / 3}} .
$$

The high-Reynolds-number analysis is now complete. An independent check on the 
analysis can be performed by recalling from section 4 that the momentum transport $M$ defined in (4.7) is the same on both walls. In terms of our asymptotic solution this implies that $r_{a} \overline{\mathcal{U}}_{a}^{\prime}(0)=-r_{b} \overline{\mathcal{U}}_{b}^{\prime}(0)$ with the wall-layer solutions $\overline{\mathcal{U}}_{a}, \overline{\mathcal{U}}_{b}$ given in (5.27), (5.33). By explicit calculation it can be shown that this is indeed the case if and only if $A_{0}$ satisfies (5.53). This gives us a high degree of confidence in the correctness of the analysis presented in this section. The corresponding expression for $M$ is

$$
M=1+R^{-1 / 6}\left\{A_{0}^{1 / 2} C_{1} \ln \left(r_{c} / r_{a}\right)+\left(\frac{\alpha c}{2}\right)^{1 / 2} r_{b} \ln (1 / \eta) \frac{\left(A_{0} P_{2}\left(r_{b}\right)\right)^{2}}{2 c^{3}}\right\},
$$

neglecting terms of $O\left(R^{-1 / 3}\right)$, from which it can be seen that the perturbation to $M$ is always positive.

For a given wavenumber $\alpha$ and radius ratio $\eta$ we can compute all the necessary flow quantities to make a direct comparison with the full Navier-Stokes solutions presented in section 4 . In order to determine quantities in the core we first solve the eigenvalue problem (5.5) to determine the pressure component $P_{2}(r)$, the wavespeed $c$ and the critical layer location $r_{c}$. The amplitude of the neutral modes follows from (5.53) with $C_{1}$ given in (5.48), and $r_{a}, r_{b}$ given in terms of $\eta$ by (2.1). The core fluctuations $A_{0} F_{2}(r) \cos \xi, A_{0} G_{2}(r) \sin \xi$ can then be calculated from the solution for $P_{2}$ using (5.4). The mean-flow distortion $\bar{u}_{1}(r)$ in the core follows from $(5.20),(5.21)$ and (5.48). The fluctuations in the wall layers are given by (5.22)-(5.25) for the inner layer, and (5.28)-(5.31) for the layer on the outer cylinder. The mean-flow distortions in the two wall layers can be calculated from (5.22), (5.27) and (5.28), (5.33). Finally, in the critical layer, we have found expressions for the first three terms $\widehat{U}_{1}, \widehat{U}_{2 L}, \widehat{U}_{2}$ in the streamwise velocity expansion in (5.35) and these are given in (5.36), (5.37) and (5.50).

\subsection{The breakdown of the asymptotic solution at small wavenumber and the emergence of the long-wave structure}

Eventually, if the wavenumber $\alpha$ is sufficiently small, the asymptotic structure set out in this section for $O(1)$ wavenumbers will become invalid and a new structure will take its place.

To see why the present structure fails, consider first the thickness of the critical layer. From (5.53) we observe that $A_{0} \sim O\left(\alpha^{-1 / 3}\right)$ as $\alpha \rightarrow 0$, which in turn means that from (5.38), the parameter $\mu \sim O\left(\alpha^{2 / 3}\right)$. It follows that the critical-layer variable $Y \sim O(\mu / \alpha)^{1 / 2} \sim O\left(\alpha^{-1 / 6}\right)$, from (5.41). Thus, in the long-wave limit, the critical-layer thickness $\sim O(\varepsilon Y) \sim O(\alpha R)^{-1 / 6}$, in view of (5.44). Turning now to the viscous wall layers, the thickness of the layer on the inner cylinder is $O\left(R^{-1 / 2} \mu_{a}^{-1}\right) \sim O(\alpha R)^{-1 / 2}$, using (5.22), (5.24). A similar argument leads to the same order-of-magnitude estimate for the thickness of the boundary-layer on the outer cylinder. Eventually, when $\alpha$ is sufficiently small, the thicknesses of the critical layer and wall layers become comparable. This occurs when $\alpha R \sim O(1)$, and implies the existence of a new larger amplitude regime in which

$$
\alpha \sim O\left(R^{-1}\right), A_{0} \sim O\left(R^{1 / 3}\right) .
$$

In this new structure the wall layer and critical layer have grown and merged to fill the whole of the annulus, so that viscous effects are now important at leading-order everywhere in the flow-field.

To determine the new scalings for the velocity and pressure we again consider the critical-layer expansions of the previous regime. From (5.35), (5.36) we see that the streamwise perturbation $\varepsilon \widehat{U}_{1} \sim \varepsilon Y$ and this becomes $O(1)$ upon using the new scalings (5.55). The corresponding radial perturbation is $\varepsilon^{2} \widehat{V}_{1} \sim \varepsilon^{2} \mu \sim O\left(R^{-1}\right)$ in the new 
regime. In addition, the pressure scales as $\varepsilon^{2} \widehat{P}_{1} \sim \varepsilon^{2} A_{0}$ and so also becomes $O(1)$. The same velocity and pressure scalings can also be deduced by considering the flow in the core or in the viscous wall layers as $\alpha \rightarrow 0$. An important extra feature in this new regime is that the leading-order pressure is independent of the radial coordinate. This can be seen most easily by appealing to the asymptotic form for $P_{2}$ in (5.17), which shows that the $r$-dependent part of the pressure $\sim O\left(\varepsilon^{2} A_{0} \alpha^{2}\right) \sim O\left(R^{-2}\right)$ in the long-wave structure. It is also worth noting that in the limit $\alpha \rightarrow 0$, the jump condition (5.34) yields $\phi \sim O\left(A_{0} \alpha^{-1 / 2}\right) \sim O\left(\alpha^{-5 / 6}\right)$, and so from (5.11) the jump in the streamwise velocity component across the critical layer $\sim \varepsilon^{2} R^{-1 / 2} \alpha^{-5 / 6}$ and therefore approaches an $O(1)$ size as $\alpha \rightarrow O\left(R^{-1}\right)$. This emphasizes the fact that, in the new regime, nonlinearity is important at leading-order throughout the flow-field and that all the harmonics of the fluctuation are of equal significance. In view of the new scaling for the wavenumber, the disturbance will now operate over a long streamwise lengthscale of $O(R)$ and evolve over an $O(R)$ timescale.

The preceding order-of-magnitude analysis now allows us to deduce the governing equations for the long-wave regime. We write

$$
[u, v, p]=\left[u^{\dagger}\left(t^{\dagger}, x^{\dagger}, r\right), R^{-1} v^{\dagger}\left(t^{\dagger}, x^{\dagger}, r\right), p^{\dagger}\left(t^{\dagger}, x^{\dagger}\right)\right]\left(1+O\left(R^{-2}\right)\right),
$$

with $x=R x^{\dagger}, t=R t^{\dagger}$. Substitution into the Navier-Stokes equations (2.3)-(2.5) yields, at leading order, the following nonlinear, viscous balances:

$$
\begin{aligned}
u_{x^{\dagger}}^{\dagger}+v_{r}^{\dagger}+r^{-1} v^{\dagger} & =0, \\
u_{t^{\dagger}}^{\dagger}+U_{B} u_{x^{\dagger}}^{\dagger}+v^{\dagger} U_{B}^{\prime}+u^{\dagger} u_{x^{\dagger}}^{\dagger}+v^{\dagger} u_{r}^{\dagger} & =-p_{x^{\dagger}}^{\dagger}\left(t^{\dagger}, x^{\dagger}\right)+u_{r r}^{\dagger}+r^{-1} u_{r}^{\dagger} .
\end{aligned}
$$

We note that the leading-order contribution from the radial momentum equation (2.5) only serves as an equation to determine the higher-order correction to the pressure and so need not be considered further. Thus, in the long-wave regime we need to solve (5.57), (5.58) subject to the no-slip conditions

$$
u^{\dagger}=v^{\dagger}=0 \text { at } r=r_{a} \text { and } r=r_{b} .
$$

The main features of these equations are the absence of streamwise diffusion, rendering them parabolic in the axial direction, and also the fact that due to the Reynolds number scaling in (5.56), the effective Reynolds number in (5.58) is unity.

The numerical solution of this long-wave problem will be discussed in section 7 .

\section{Comparison of asymptotic and numerical results}

In the previous section we proposed an asymptotic form for the nonlinear instability of annular sliding Couette flow. Now we wish to compare this solution with our NavierStokes computations from section 4. In particular we are interested in (i) how large the Reynolds number needs to be to obtain reasonable agreement between the solutions, (ii) which of the modes discovered in section 3 does the asymptotic solution best approximate and (iii) is it possible to see the delicate asymptotic flow structure in the finite Reynolds number computations?

First, from numerical computation of the Rayleigh equation (5.5) we find that there is a unique value $y_{c}=-0.9866$ at $(\eta, \alpha)=(0.1,0.6546)$ for which $A_{0}$ in $(5.53)$ is finite. It is therefore clear immediately from figure 2 (a) that the asymptotic theory is a better approximation to the lower branch than the upper branch. A close-up version of figure 2(a) for the lower branch at high $R$ is shown in figure 9(a), together with the corresponding asymptotic result. In the figure, we can see that the agreement between the numerical 
(a)

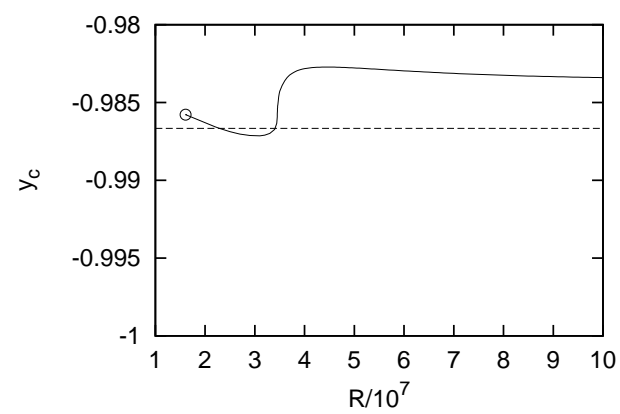

(b)

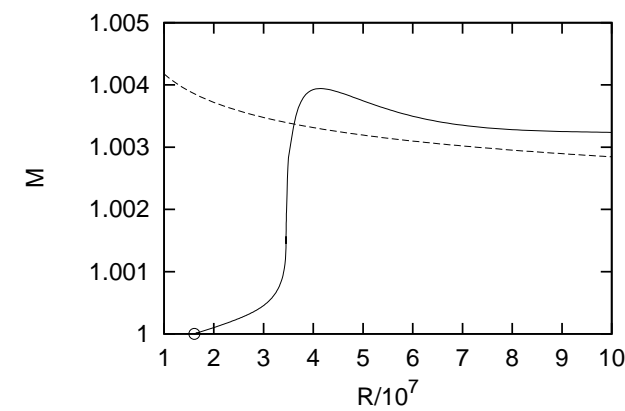

Figure 9. The solid lines represent the lower branch solution for $(\eta, \alpha)=(0.1,0.6546)$. The truncation level $(L, N)=(240,100)$ is used. The dashed lines are the asymptotic results $y_{c}=-0.9866$ and $M=1+0.0613 R^{-1 / 6}$. Open circles in the figures represent linear critical points.

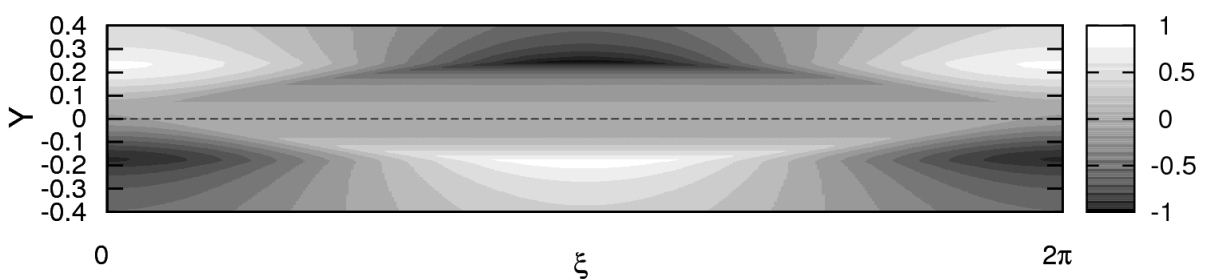

Figure 10. The flow structure in the vicinity of the critical layer for $(\eta, \alpha)=(0.1,0.6546)$. The grey scale represents contours of $\widetilde{\hat{U}}_{2 Y}$.

and asymptotic solutions is good once the kink at $R=3.5 \times 10^{7}$ has been passed. In figure 9 (b) we compare the corresponding values of the momentum transport $M$. Again, it can be seen that after the kink, the solution branch is approximated reasonably well by the asymptotic result.

Encouraged by the agreement obtained thus far, we now seek to compare the numerically and asymptotically determined flow structures. For this purpose, we make the comparisons for each layer by using only the leading order asymptotic solutions as follows.

First we consider the nonlinear critical layer structure in terms of the stretched radial coordinate $Y=\varepsilon^{-1}\left(y-y_{c}\right)$. The asymptotic critical layer solution for the streamwise velocity has the expansion

$$
u=\varepsilon \widehat{U}_{1}(Y)+\varepsilon^{2} \ln \varepsilon \widehat{U}_{2 L}(\xi)+\varepsilon^{2}\left\{\widetilde{\hat{U}}_{2}(\xi, Y)+\widehat{\hat{U}}_{2}(Y)\right\}+\cdots,
$$

where $\widehat{U}_{2}(\xi, Y)$ is numerically decomposed into the fluctuation $\widetilde{\hat{U}}_{2}(\xi, Y)$ and the mean $\widehat{\hat{U}}_{2}(Y)$. For the sake of clarity, the fluctuation is differentiated with respect to $Y$, and the result is shown in figure 10. We compare this with the corresponding numerical result $\varepsilon^{-2} \tilde{u}_{Y}(\xi, Y)$ (figure 11). One of the characteristic structures associated with the asymptotic solution $\widetilde{\hat{U}}_{2 Y}$ is the cats-eye shape which possesses, at this order, a discontinuity in the first derivative at $Y-r_{c} \bar{u}_{1}\left(r_{c}\right) \tau_{0}^{-1}= \pm\left(2 r_{c} \mu(1-\cos \xi) / \alpha \tau_{0}\right)^{1 / 2}$. We can detect a similar quasi-discontinuous structure in the high $R$ numerical solutions (P1 and P6). However, when $R$ is smaller than its value at the kink, the discontinuity seems to be smoothed out due to the thicker nonlinear critical layer structure which effectively overlaps with the inner wall layer. For example, the visualization for P1 and P2 is similar at first glance, but there is only an upper (outer) discontinuity for P2 whereas we can see a 
P1: $R=2 \times 10^{6}$ upper branch

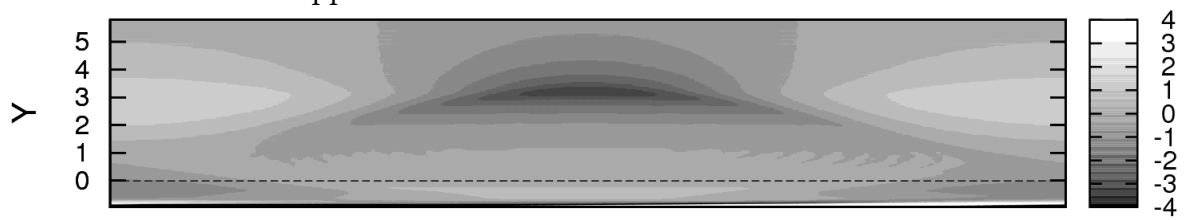

P2: $R=2 \times 10^{5}$ upper branch

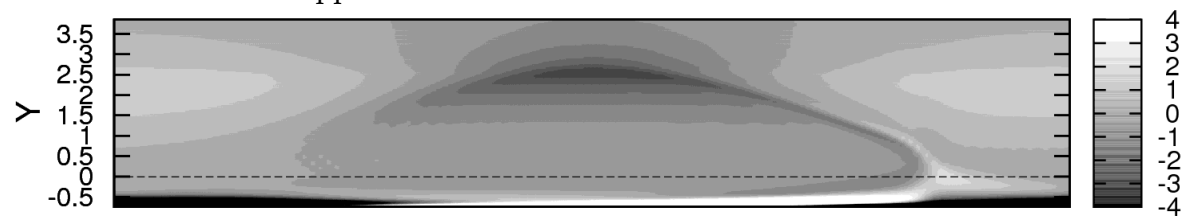

P3: $R=2 \times 10^{5}$ lower branch

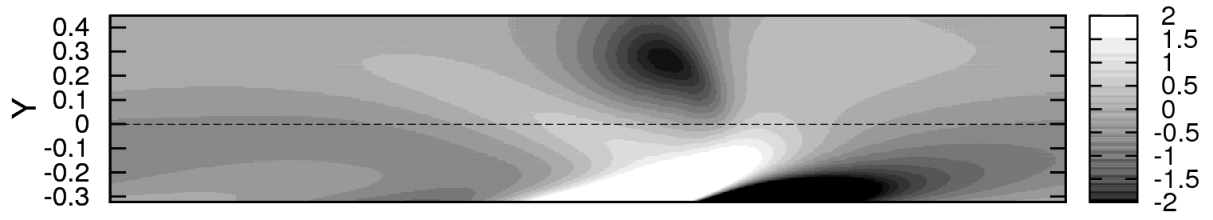

P4: $R=2 \times 10^{6}$ lower branch

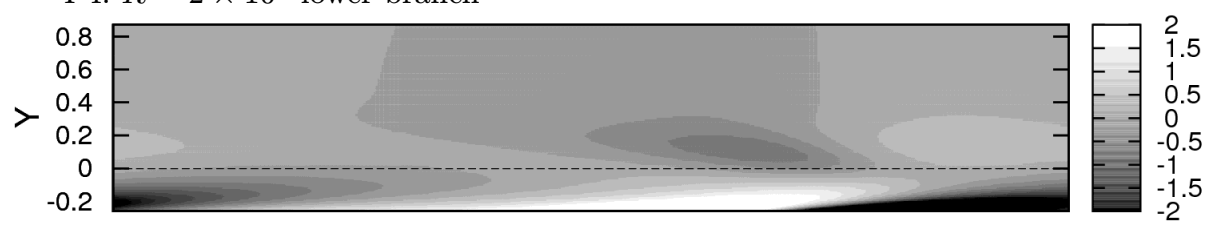

P5: $R=2 \times 10^{7}$ lower branch

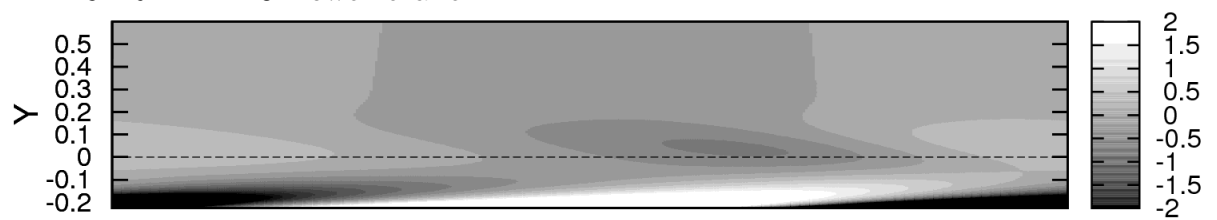

P6: $R=10^{8}$ lower branch

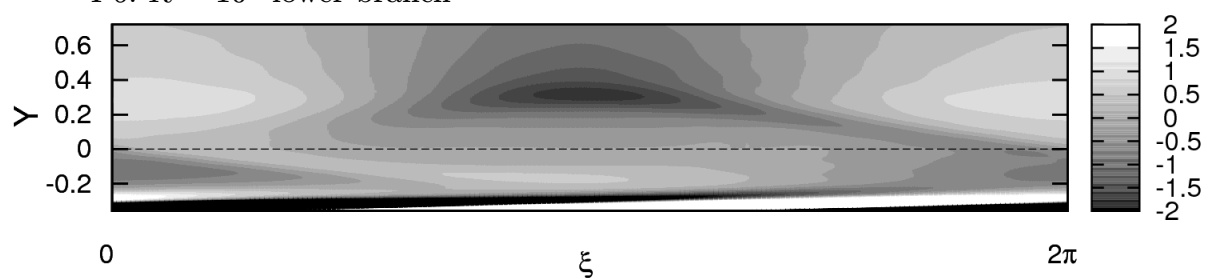

Figure 11. The flow structure in the vicinity of the critical layer for $(\eta, \alpha)=(0.1,0.6546)$. The grey scale represents contours of $\varepsilon^{-2} \tilde{u}_{Y}$. The radial plot intervals are the same as the close-up plots in figures 3 and 4 .

lower (inner) discontinuity for P1. In other words the kink, which differentiates between the LR and HR modes, can be considered to be the product of the separation of the nonlinear critical layer and the inner wall layer structures. This feature is reminiscent of the role of the kink investigated by Healey (1995) for the linear stability of the Blasius boundary layer, but seen here in a nonlinear setting.

Next we compare asymptotic and numerical streamwise velocity solutions in the core 
(a)

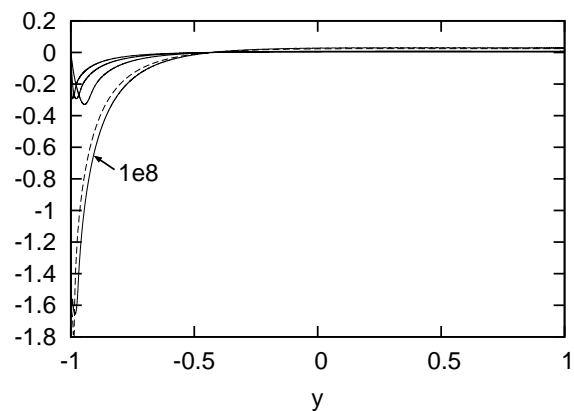

(c)

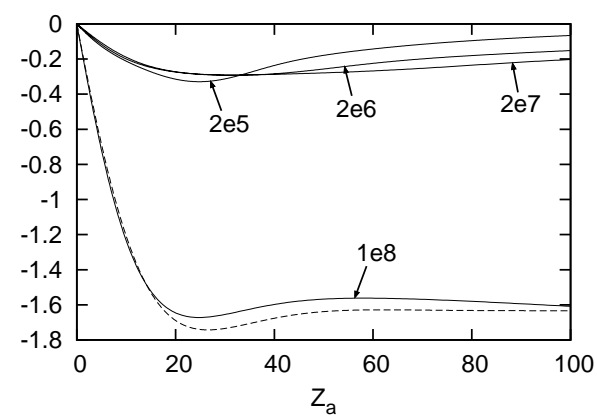

(b)

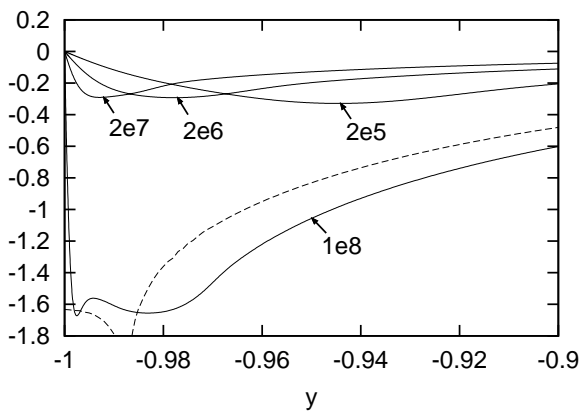

(d)

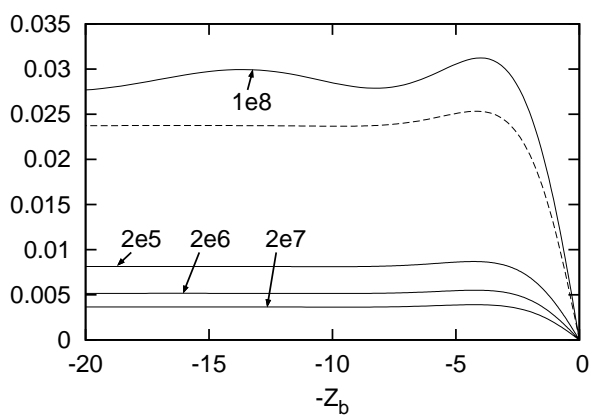

FIGURE 12. The comparison of the real part of the fluctuation $F$ for the lower branch finite-amplitude solutions (solid lines, $\varepsilon^{-2} F$ ) and the asymptotic results (dashed lines, $A_{0} F_{2}$ for (a) and (b), $\mathcal{F}_{a}$ for $(\mathrm{c}), \mathcal{F}_{b}$ for $\left.(\mathrm{d})\right)$ for $(\eta, \alpha)=(0.1,0.6546)$. The values of $R=\mathrm{A} \times 10^{\mathrm{N}}$ are abbreviated as AeN.

and the wall layers, restricting our attention to the lower branch numerical solution. The asymptotic core solution for the streamwise component expands in the form

$$
u=\varepsilon \bar{u}_{1}(y)+\varepsilon^{2}\left\{A_{0} F_{2}(y) \cos \xi+\bar{u}_{2}(y)\right\}+\cdots,
$$

while the inner wall layer solution is written in terms of functions of $Z_{a}=R^{1 / 2}(1+y)$ as

$$
u=\varepsilon^{2}\left\{\frac{1}{2} \mathcal{F}_{a}\left(Z_{a}\right) e^{\mathrm{i} \xi}+\text { c.c. }\right\}+\varepsilon^{4} \overline{\mathcal{U}}_{a}\left(Z_{a}\right)+\cdots
$$

and the outer wall layer solution is expressed in terms of the variable $Z_{b}=R^{1 / 2}(1-y)$ as

$$
u=\varepsilon^{2}\left\{\frac{1}{2} \mathcal{F}_{b}\left(Z_{b}\right) e^{\mathrm{i} \xi}+\text { c.c. }\right\}+\varepsilon^{4} \overline{\mathcal{U}}_{b}\left(Z_{b}\right)+\cdots
$$

Therefore we see that the axial-dependence in the leading-order terms of the asymptotic solution in both the core and the wall layers consists of just a single harmonic. We recall that the numerical solution for the streamwise velocity takes the form

$$
u=\bar{u}(y)+\left\{\frac{1}{2} F(y) e^{\mathrm{i} \xi}+\text { c.c. }\right\}+\text { higher order Fourier modes. }
$$

The real and imaginary parts of $\varepsilon^{-2} F$ and $A_{0} F_{2}, \mathcal{F}_{a}, \mathcal{F}_{b}$ are compared in figures 12 and 13 respectively. Note that the real and imaginary part of $F$ corresponds to the even $(\cos \xi)$ and odd $(\sin \xi)$ axial dependence respectively. In the outer part of the core, above the critical layer, both asymptotic and numerical solutions predict that $F$ is even, to leading order. In the inner core, where the asymptotic theory again predicts an even stream- 
(a)

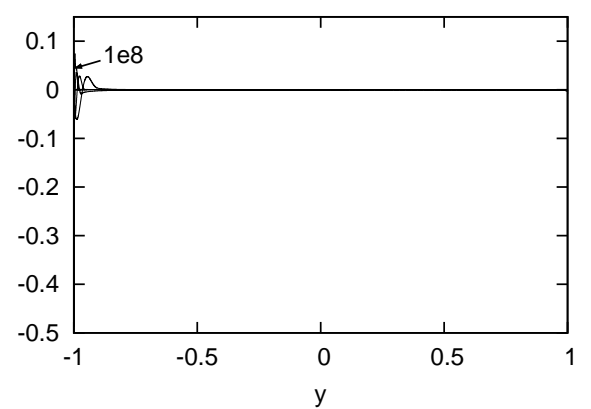

(c)

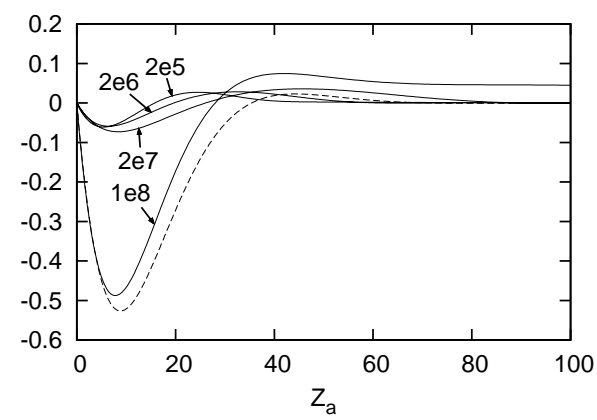

(b)

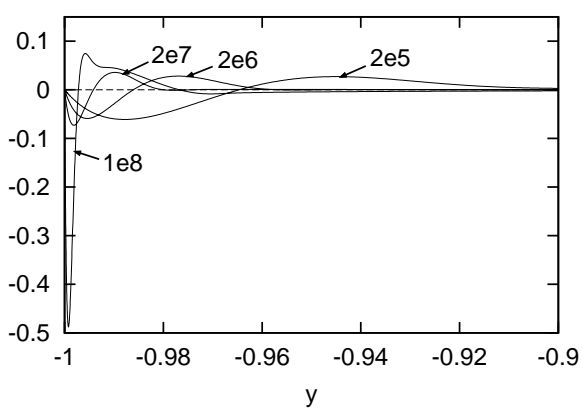

(d)

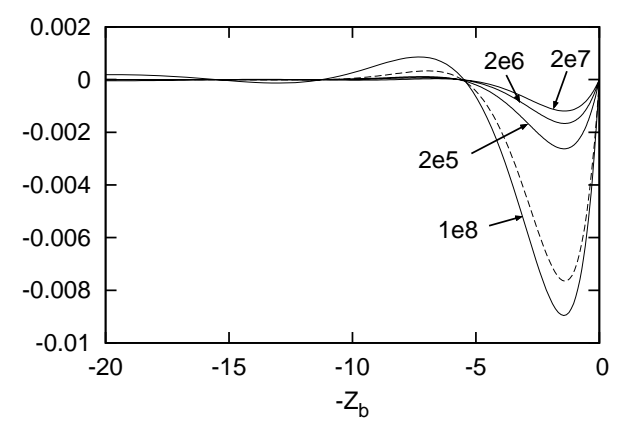

Figure 13. Caption as for figure 12 except that the imaginary parts are now compared.

wise velocity, such agreement is harder to obtain, in part due to the interaction of the nonlinear critical layer and inner wall layer referred to earlier. Except for this difference, the numerical solutions qualitatively agree with the asymptotic result. Furthermore, the solutions begin to show quantitative agreement after the kink is passed, as predicted in figure 9 . The two peaks of $F$ for $R=10^{8}$ in figure 12 (b) also suggest that the separation of the inner wall layer and the nonlinear critical layer is underway.

The mean-flow distortion is also compared in figure 14. Because the leading order magnitude of the asymptotic solutions is different in each layer, we compare $\varepsilon^{-1} \bar{u}$ and $\bar{u}_{1}$ for the core region and $\varepsilon^{-4} \bar{u}$ and $\overline{\mathcal{U}}_{a}, \overline{\mathcal{U}}_{b}$ for the wall layers. Again, good qualitative and quantitative agreement of asymptotic and numerical solutions can be found before and after the kink. There are two prominent features close to the inner wall. One is the sharp peak, which is related to the asymptotic discontinuity of the core solution at the critical layer. The other is the negative perturbation to the mean flow near the inner wall which arises in order to maintain the same value of $M$ at the inner and outer walls. A plot of the normalized nonlinear forcing

$$
N_{f}(y) \equiv(\alpha R) r^{-1} \partial_{r}\left\{r \overline{\psi_{\xi}\left(\psi_{r}+r^{-1} \psi\right)}\right\}=-r^{-1} \partial_{r}\left(r \bar{u}^{\prime}\right)
$$

calculated from the numerical solution (figure 15), reveals that the mean-flow distortion is almost unforced in the outer core, as predicted by asymptotic theory.

\section{The behaviour of the solutions as $\alpha \rightarrow 0$}

Here we focus on LR mode solutions which, we recall, do not show quantitative agreement with the asymptotic analysis of section 5 due to the incomplete nature of the nonlinear critical layer structure (see, for example figure 11). One of the reasons that 
(a)

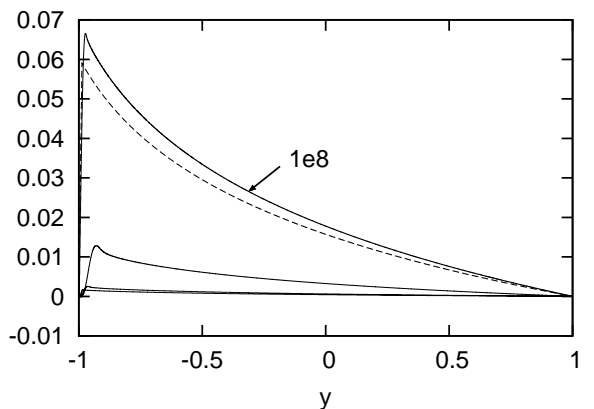

(c)

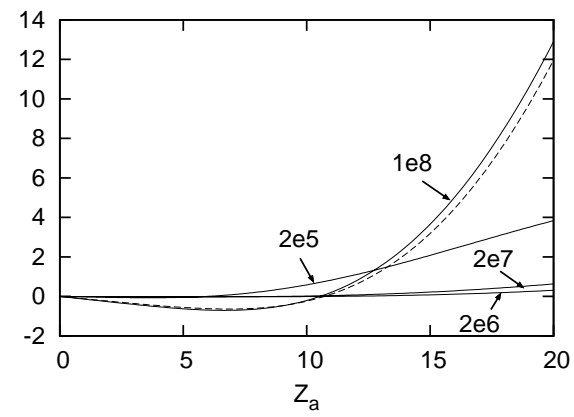

(b)

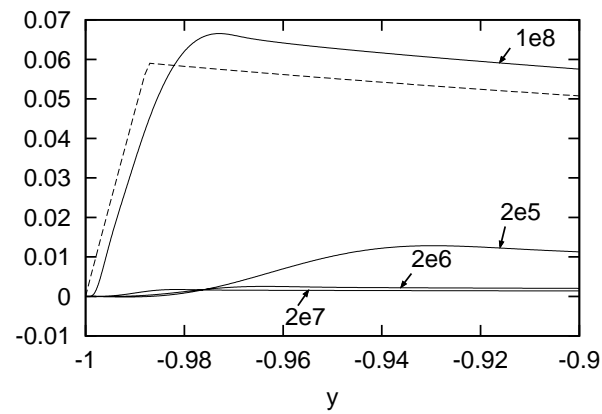

(d)

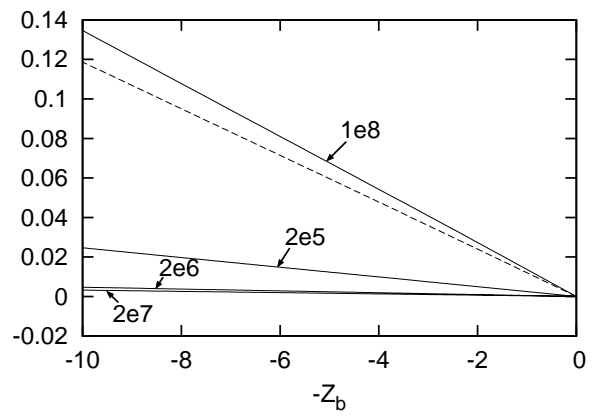

FiguRE 14. The comparison of the mean-flow distortion for the lower branch finite-amplitude solutions (solid lines, $\varepsilon^{-1} \bar{u}$ for (a) and (b), $\varepsilon^{-4} \bar{u}$ for (c) and (d)) and the asymptotic results (dashed lines, $\bar{u}_{1}$ for (a) and (b), $\overline{\mathcal{U}}_{a}$ for (c), $\overline{\mathcal{U}}_{b}$ for $\left.(\mathrm{d})\right)$ for $(\eta, \alpha)=(0.1,0.6546)$. The values of $R=\mathrm{A} \times 10^{\mathrm{N}}$ are abbreviated as AeN.

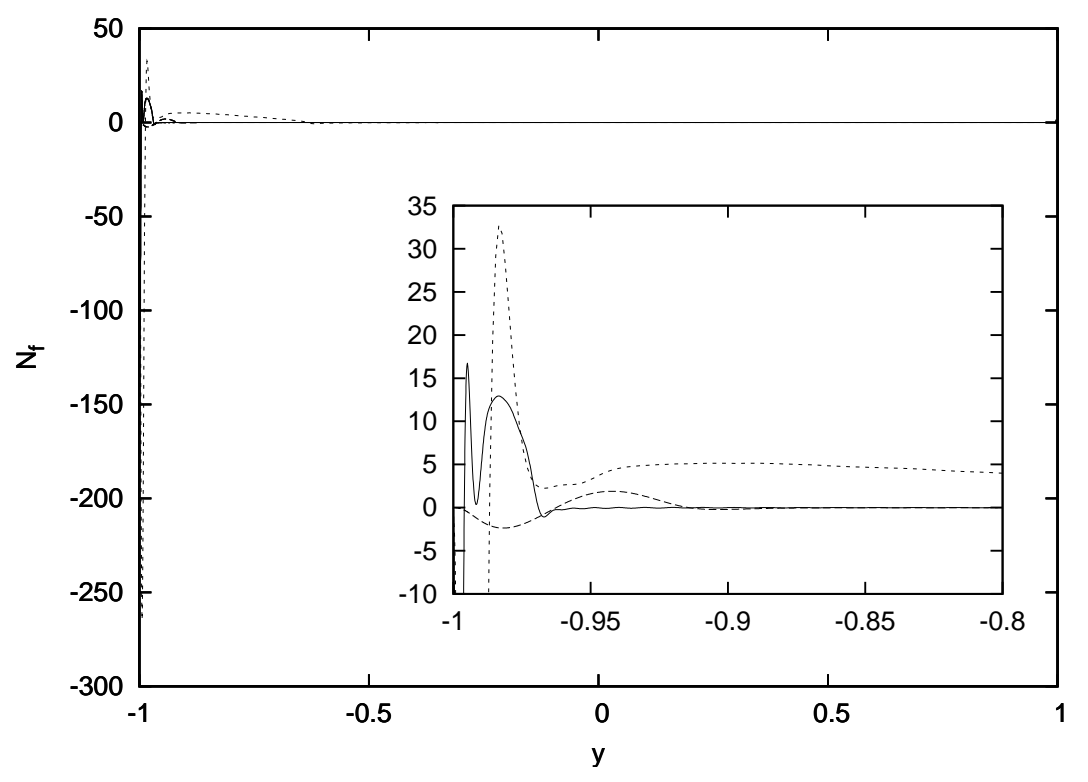

FIgURE 15. The nonlinear forcing of the mean-flow distortion for $(\eta, \alpha)=(0.1,0.6546)$. The solid, dashed and dotted lines correspond to P6 $\left(R=10^{8}\right.$, lower branch), P3 ( $R=2 \times 10^{7}$, lower branch), $\mathrm{P} 1\left(R=2 \times 10^{6}\right.$, upper branch) respectively. 
(a): $\alpha=0.05$

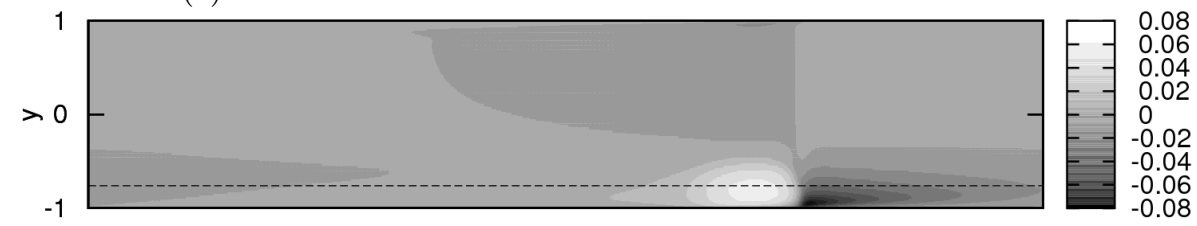

(b): $\alpha=0.02$

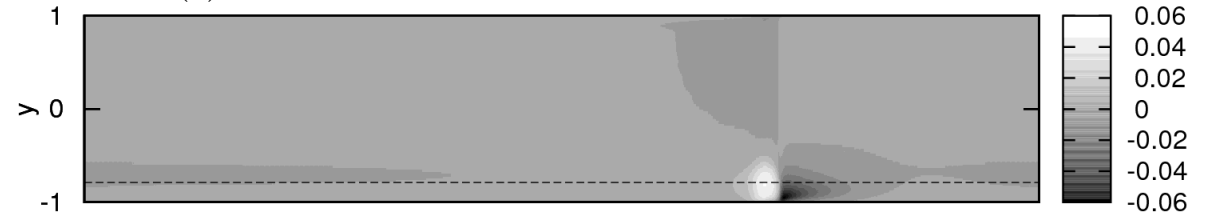

(c): $\alpha=0.01$

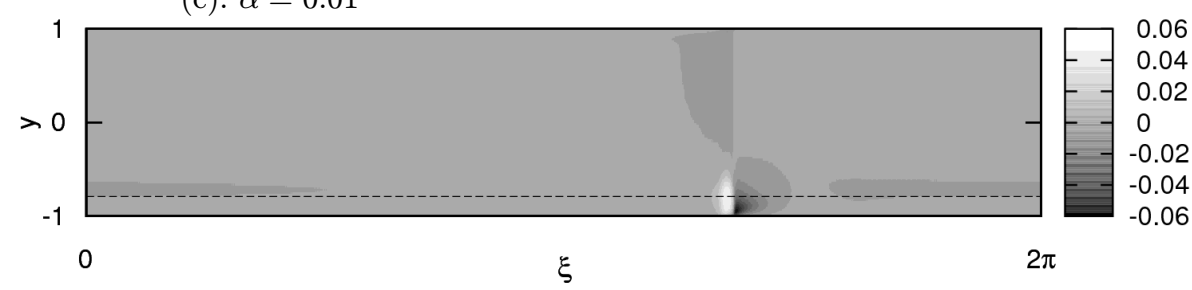

Figure 16. The axial fluctuation velocity field $\tilde{u}$ for $(\eta, R)=\left(0.33,5 \times 10^{4}\right)$. The resolution $(L, N)=(50,240)$ is used for $(\mathrm{a})$ while $(L, N)=(30,500)$ is used for (b) and (c). The dashed line represents the location of the critical layer.
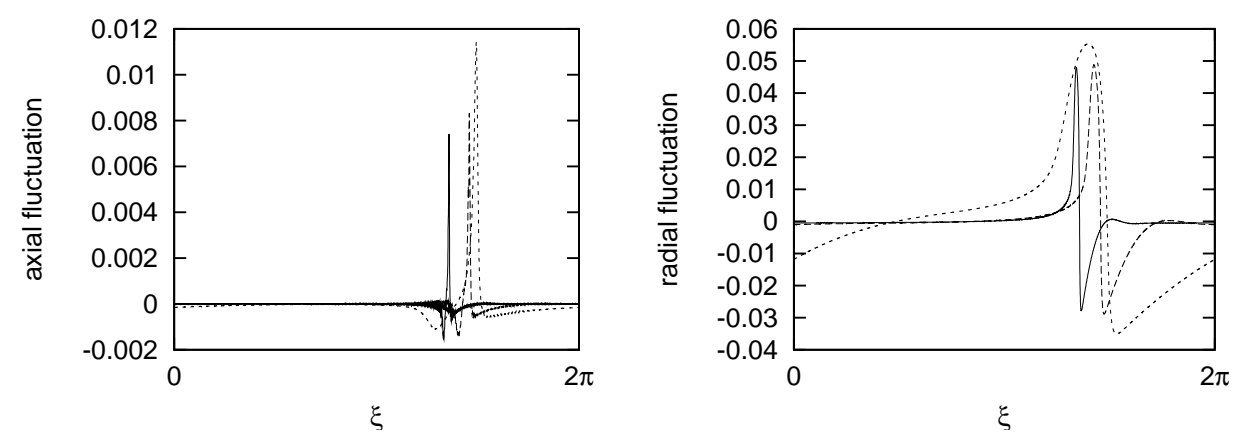

FIgURE 17. The fluctuation velocity field at the critical layer location $y=y_{c}$ for $(\eta, R)=(0.33$, $\left.5 \times 10^{4}\right)$. Left and right figures represent $\tilde{u}$ and $\tilde{v}$. The solid, dashed and dotted curves correspond to $\alpha=0.01,0.02$ and 0.05 respectively. The truncation level is the same as for figure 16 .

this limit is of interest is that numerical evidence suggests that the maximum value of $\eta, \eta_{\max }$ say, beyond which solutions do not exist, occurs as $\alpha \rightarrow 0$. In this section we will first study the behaviour of the solutions of the full Navier-Stokes equations at fixed Reynolds number and radius ratio when $\alpha$ is small but finite. We will then continue the solution branch in the limit $\alpha \rightarrow 0$ by studying the reduced (long-wave) problem that emerges when $\alpha \sim O\left(R^{-1}\right)$.

From examination of figure 7 , it appears that $\eta_{\max }$ tends to a maximum limiting value in the range $0.33-0.40$ as $\alpha \rightarrow 0$. A more accurate estimate than this is difficult to obtain due to the high resolution required. In view of this, we choose to examine the behaviour as $\alpha \rightarrow 0$ by fixing $\eta=0.33, R=5 \times 10^{4}$. The fluctuation component of the streamwise velocity is visualized in figure $16(\mathrm{a})-(\mathrm{c})$ for $\alpha=0.05,0.02$ and 0.01 . These 
(a)

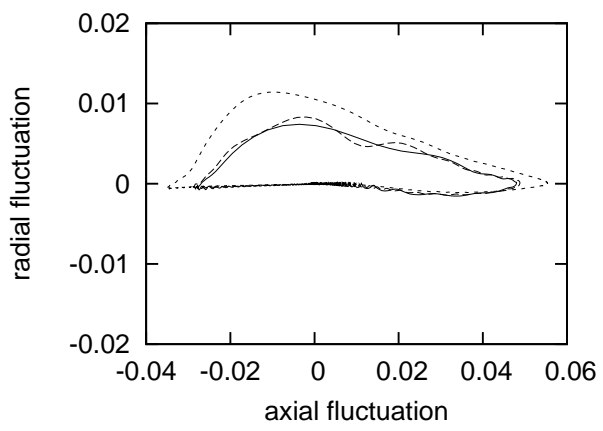

(b)

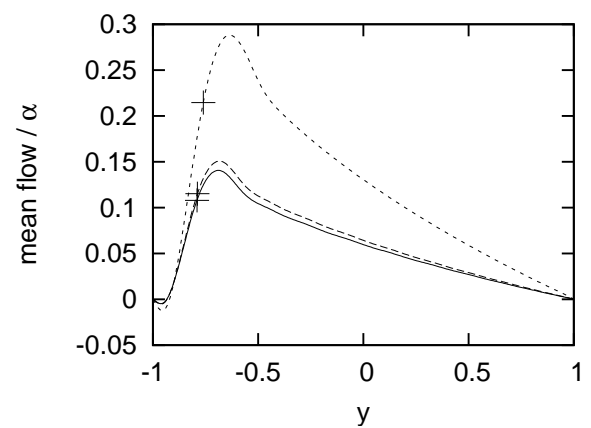

FiguRE 18. The convergence of the flow field as the wavenumber $\alpha$ is decreased for $(\eta, R)=(0.33$, $\left.5 \times 10^{4}\right)$. The truncation level is the same as for figure 16. (a): Plot of flow field along the axis in the phase space spanned by $\tilde{u}$ and $\tilde{v}$ for $\xi \in[0,2 \pi]$ at the critical layer location $y=y_{c}$. (b): The normalized mean flow $\bar{u} / \alpha$. The solid, dashed and dotted curves correspond to $\alpha=0.01,0.02$ and 0.05 respectively. The crosses indicate the critical layer locations.

figures show an intriguing localization of the disturbance in the streamwise direction. To see the localization more clearly, the fluctuation field at the critical level $y=y_{c}$ is shown in figure 17 where we can see the concentration of both the axial and radial velocities in the localized region.

For the reader who is interested in the spectral convergence properties of the localized solution, we remark in passing that the shape of this localized structure does not change when the resolution level is increased with $\eta$ below the cut-off value (which lies in the range $0.33-0.40$ as mentioned previously). If a lower resolution level is used however, it is possible to continue our branch beyond this threshold; however, these solutions are sensitive to spectral accuracy and cease to exist once the resolution level is improved. The latter property of our solution is similar to that for poorly-resolved localized solutions observed in plane Couette flow by Cherhabili \& Ehrenstein (1995); see Rincon (2007), Ehrenstein et al. (2008), Mehta (2004) and Mehta \& Healey (2005) for more discussion of this issue. Thus, we conclude that our existence threshold of $\eta$ for the LR mode is reliable.

The smaller the value of $\alpha$, the stronger the localization becomes and it appears that the shape of the fluctuation is converging to a limiting form as $\alpha \rightarrow 0$. To show this tendency more clearly, the plots of $\tilde{u}$ and $\tilde{v}$ are combined in figure 18(a). The mean-flow distortion for the different values of $\alpha$ is compared in figure 18(b) and we see that this quantity scales with $\alpha$ when $\alpha$ is small. This makes sense because the mean-flow distortion is sustained by the spatial average of the nonlinear self-interaction of the fluctuation (see (4.3)) and therefore if the shape of the fluctuation is becoming independent of $\alpha$, the mean-flow distortion divided by $\alpha$ must converge to a limiting form. However when $\alpha$ is sufficiently small, specifically of $O\left(R^{-1}\right)$, the solution cannot continue to exist in its present form at finite $R$ as it would violate the energy analysis of section 3 which, for $\eta=0.33$, shows that all disturbances must monotonically decay if $\alpha R_{E} \lesssim 108.20$.

The new structure that controls the behaviour of the modes at very small $\alpha$ is the longwave version of the asymptotic structure of section 5 , and the governing equations are (5.57)-(5.58). Indeed, in figure 16, we can see that the nonlinear critical layer structure we saw in figure 11 is completely smoothed out as we discussed in subsection 5.4.

The strategy we adopt to obtain the solution of $(5.57)-(5.58)$ is the continuation of the solution branch of (4.2)-(4.3), gradually decreasing $\alpha$, while keeping the quantity 
(a)

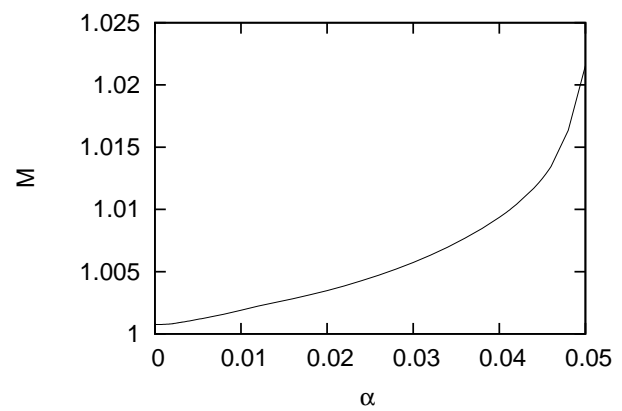

(b)

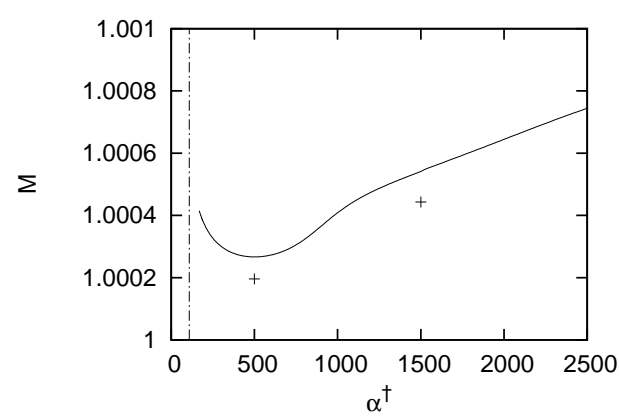

Figure 19. (a): The continuation to the long-wave limit for $\alpha^{\dagger} \equiv R \alpha=2500$. (b): The solid line shows the solution branch of the long-wave limit problem. The vertical dash-dotted line represents the energy threshold, calculated from the analysis of section 3 . The truncation level $(L, N)=(50,240)$, which is sufficient to see the tendency of the branch, is used. Crosses are the same solution but computed at a higher resolution level, i.e. $(L, N)=(50,400)$ is used for $\alpha^{\dagger}=1500$ while $(L, N)=(40,500)$ is used for $\alpha^{\dagger}=500$.

(a): $\alpha^{\dagger}=1500$

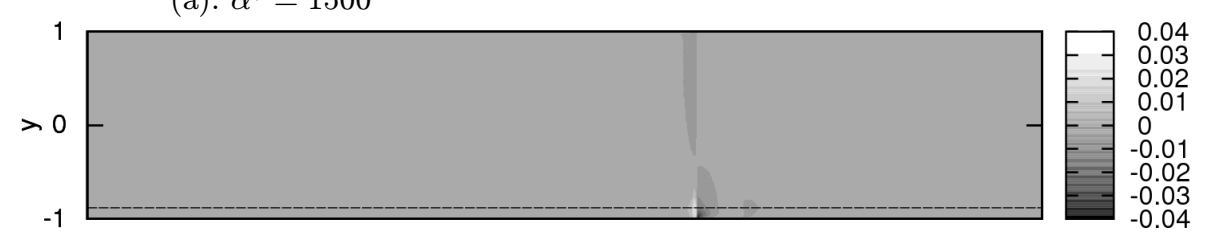

(b): $\alpha^{\dagger}=500$

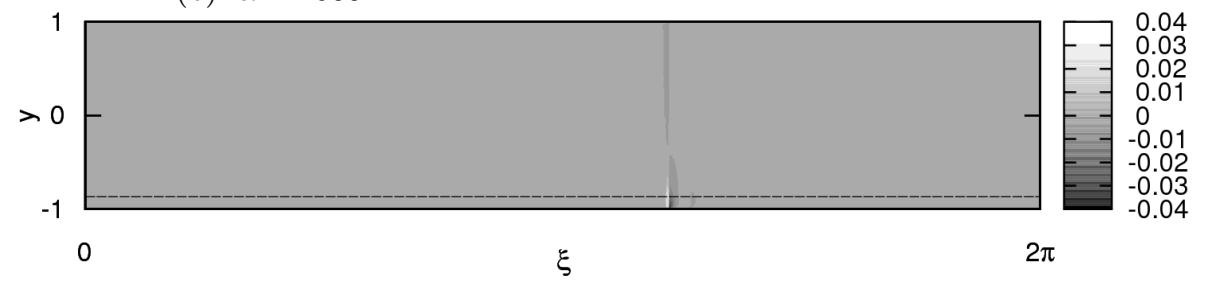

FIgURE 20. The axial fluctuation velocity field $\tilde{u}^{\dagger}$ for the long-wave limit solution at a radius ratio $\eta=0.33$. The resolution $(L, N)=(50,400)$ is used for $\alpha^{\dagger}=1500$, while $(L, N)=(40,500)$ is used for $\alpha^{\dagger}=500$. The dashed line represents the location of the critical layer.
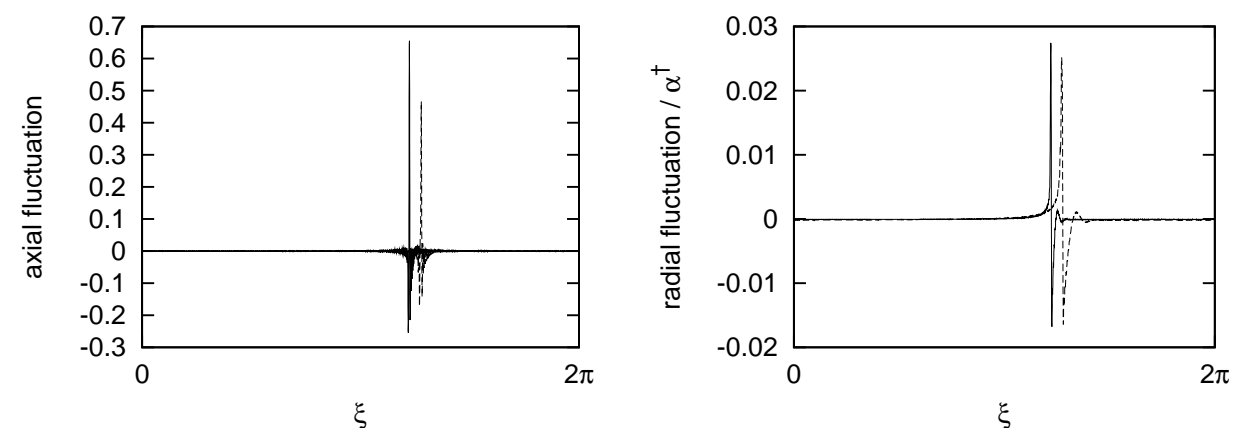

Figure 21. The fluctuation velocity field at the critical layer location $y=y_{c}$ for $\eta=0.33$. The left figure represents $\tilde{u}^{\dagger}$ whereas the right figure represents $\tilde{v}^{\dagger} / \alpha^{\dagger}$. The solid and dashed curves correspond to $\alpha^{\dagger}=500$ and 1500 respectively. The truncation level is the same as for figure 20 . 
$\alpha^{\dagger} \equiv \alpha R$ constant. Once the solutions for $\psi(\xi, y)$ and $\bar{u}(y)$ are obtained, $\left[u^{\dagger}, v^{\dagger}\right](\xi, y)$ can be found as $\left[\bar{u}+\psi_{r}+r^{-1} \psi,-\alpha^{\dagger} \psi_{\xi}\right]$. The continuation is started from the lower branch solution at $(\eta, \alpha, R)=\left(0.33,0.05,5 \times 10^{4}\right)$ as shown in figure $19(\mathrm{a})$. The solution branch successfully reaches $\alpha=0$, allowing us to then compute the bifurcation curve shown in figure 19(b) by varying $\alpha^{\dagger}$. The solution branch reaches a minimum in $M$ at $\alpha^{\dagger} \simeq 500$ and then further decrease in $\alpha^{\dagger}$ leads to a rapid increase in $M$, thereby avoiding crossing the energy boundary calculated in section 3 and shown as a vertical dot-dashed line on figure 19(b). As expected, the solutions of the long-wave problem possess highly streamwiselocalized fluctuations with no discernible critical layer structure (figures 20 and 21) and there is good qualitative agreement with the finite Reynolds number calculations shown in figure 16. The strong localization observed here clearly requires a large number of axial modes $N$ for satisfactory resolution. However, the lack of an obvious critical layer means that the solution can be fully-resolved in the radial direction without $L$ needing to be excessively large. We have exploited this fact when producing figure 19(b) where we have lowered $L$ and increased $N$ to calculate the solution marked with a cross for $\alpha^{\dagger}=500$.

\section{Conclusion}

In this paper we have examined the large Reynolds number asymptotic behaviour of two-dimensional finite-amplitude travelling-wave solutions of shear flows. For this purpose, we extended the axisymmetric solution branch of annular sliding Couette flow computed by DN11 and developed a corresponding axisymmetric asymptotic theory valid at high Reynolds number.

The initial finite-amplitude computation was conducted at a radius ratio $\eta=0.1$, where we can find linear instability, and we fixed the wavenumber $\alpha$ at its linear critical value of 0.6546. Finite-amplitude solutions come into existence due to a saddle-node bifurcation, and upper and lower branches in amplitude-Reynolds number space are formed. Both branches continue to exist as $R$ is increased, although the lower branch coincides with the linear instability, i.e. the zero-amplitude state, for a finite range of $R$. At sufficiently high $R$, our calculations indicated that there exist kinks in both the upper and lower branches. The part of each branch before and after the kink is referred to as the LR (low Reynolds number) and HR (high Reynolds number) mode respectively.

A multi-structured analytic solution valid for asymptotically large $R$ was derived in section 5: this asymptotic theory divides the shear layer into five decks (figure 8). At leading order viscosity is only present in the boundary layers near the inner and outer walls and is also vital in the nonlinear critical layer where a cats-eye shape quasi-singularity exists and the mean flow is strongly modified. The asymptotic theory was then compared with the finite Reynolds number calculations. For the LR mode, where $R$ is in the range $O\left(10^{4}\right)-O\left(10^{7}\right)$, we observed good qualitative agreement between the asymptotic solutions for the various layers and the corresponding visualization of the numerical solutions. However, for this mode, the inner inviscid core layer is absent and hence the nonlinear critical layer structure is incomplete, due to the effective interaction with the inner wall layer. The reason for this is that the distance between the nonlinear critical layer and the inner wall layer is so close that it is comparable with the thickness of the critical layer, which is of order $R^{-1 / 6}$ according to the theory. When we further increase $R$ to $10^{8}$, so that the relevant solution branch belongs to the HR mode, we find that the flow visualization of the lower branch solution agrees quantitatively with the asymptotic solution, with visualizations showing a clear separation of the inner wall layer and the critical layer. Therefore we conclude that the kink in the solution branch results from the 
separation of the inner wall layer and the nonlinear critical layer. In the linear setting, Healey (1995) came to a similar conclusion when considering boundary-layer stability.

One of the notable properties of this asymptotic solution, and one which agrees with the numerically-computed lower branch asymptotic behaviour, is that all disturbances decay as $R$ is increased. This means that when we consider practical laminar flow control at high $R$, the present Tollmien-Schlichting type flow structure could become more important than the vortex-wave interaction type streamwise roll equilibrated states, which require an $O(1)$ modification of the mean flow, because it would be 'closer' to laminar flow in the sense of disturbance magnitude (e.g. disturbance norm or energy). Thus it is likely that our solution opens up the route to transition first in some cases. We also note here that for moderate $R$, it is known that an $O\left(R^{-1}\right)$ streamwise roll can trigger shearflow transition by the lift-up mechanism (e.g. Lemoult et al. 2012). When $R$ is sufficiently large, our solutions, which have $O\left(R^{-1 / 6}\right)$ deviation from the basic flow, would also affect this route to transition where the magnitude of the disturbance evolves from $O\left(R^{-1}\right)$ to an $O(1)$ size.

As we have already remarked in the introduction, one of the possible practical applications of annular sliding Couette flow would be to the boundary-layer flow surrounding an object in flight. At first, one might be concerned that the values of Reynolds number $R$ considered in this paper are too large to apply to this real situation. However, we note here that the length scale must be chosen as the inner cylinder radius in order to make the comparison. Thus when the results of annular sliding Couette flow with $\eta=0.1$ are applied, we find that the Reynolds number, $R_{f}$ say, determined by the radius and speed of the flying body is given by $R_{f}=2 R \eta /(1-\eta)=0.22 R$, and therefore stays at a realistic value. Obviously the smaller the value of $\eta$, the smaller the corresponding value of $R_{f}$. We also note that our results demonstrate that the main activity in the flow-field is localized near the inner wall so that the effective boundary-layer thickness is significantly less than the gap width.

We also examined how the finite-amplitude solution branch behaved as $\eta$ is varied. It is found that the solution branches of both the HR and LR mode can be continued well beyond the linear long-wave cut-off value of $\eta \simeq 0.1415$. For the LR mode, the nonlinear cut-off value of $\eta$ increases as $\alpha$ decreases. The ultimate value for small $\alpha$ is estimated to lie between 0.33 and 0.40 . This observation suggests that the nonlinear version of the cut-off is also a long-wave phenomenon. Although the present calculation failed to trace the HR mode solution branch beyond a value of $\eta \simeq 0.159$, it must also reach a cut-off if we assume that this mode asymptotes according to the large $R$ theory which must itself experience a cut-off before the narrow gap limit of plane Couette flow is reached.

The viscous layers in the asymptotic structure become thinner as $R$ is increased, but thicken as $\alpha$ is decreased. A distinguished limit $\alpha \sim O\left(R^{-1}\right)$ arises at which the wall layers and critical layer thicken to an $O(1)$ size and merge together. An identical effect is observed in the numerical solutions where we observe that as $\alpha$ is decreased to 0.01 for $\eta=0.33$, the rapidly-varying behaviour near the critical location is completely smoothed out. Instead, there is the formation of a prominent streamwise localized structure. This localized structure can be explained by the $\alpha \sim O\left(R^{-1}\right)$ theory because all streamwise Fourier modes become leading order in size, in contrast to the asymptotic theory for $\alpha \sim O(1)$. In order to check this argument, we examined numerically the limit $\alpha \rightarrow 0$ of the finite-amplitude solution branch, while keeping the product $\alpha R$ fixed. In this limit, the governing equations coincide with the $\alpha \sim O\left(R^{-1}\right)$ version of the asymptotic equations. The visualization of the corresponding solution indeed shows strong localization. The retreat of the solution to high Reynolds number in the small wavenumber limit is also necessary so that the solutions do not cross the energy boundary derived in section 3 . 
Although the axisymmetric solutions presented here appear to have some connection to the initiation of localized turbulent spots, it should be noted that the present solutions cannot be linked directly to fully developed 'puffs' in pipe flow, because the axisymmetric asymptotic solution presented in this study does not exist in this flow (as remarked at the beginning of section 5). Instead, by analogy with our results, it might be expected that a more relevant asymptotic model for that situation would be the spiral wave structure given by Smith \& Bodonyi (1982a), and in particular its long-wave limit, which gives a threshold amplitude for the instability.

This work was partially supported by the JSPS Institutional Program for Young Researcher Overseas Visits. K.D. would like to thank Professors P. Hall and R. R. Kerswell for inspiring discussions concerning localized solutions.

\section{Appendix A. The phase shift calculation for the asymptotic solution}

Here our aim is to calculate the jump in the $\sin \xi$ component of axial velocity across the critical layer considered in section 5.3. It is shown there that the term $\widehat{U}_{5}(\xi, Y)$ in (5.35) is the largest to possess an odd part about $\xi=\pi$. We therefore write

$$
\widehat{U}_{5}=\widehat{U}_{5 \mathrm{O}}+\widehat{U}_{5 \mathrm{E}},
$$

with subscripts $\mathrm{O}$ and $\mathrm{E}$ to denote the odd and even parts of this expression, and we do likewise for the corresponding radial velocity and pressure components. From substitution of (5.35) into the Navier-Stokes equations $(2.3)-(2.5)$, we find that $\left(\widehat{U}_{5 \mathrm{O}}, \widehat{V}_{5 \mathrm{E}}, \widehat{P}_{5 \mathrm{O}}\right)$ are governed by

$$
\begin{aligned}
\alpha \widehat{U}_{5 \mathrm{O} \xi}+\widehat{V}_{5 \mathrm{E} Y} & =0 \\
\alpha \widehat{U}_{1} \widehat{U}_{5 \mathrm{O} \xi}+\widehat{V}_{1} \widehat{U}_{5 \mathrm{O} Y}+\widehat{V}_{5 \mathrm{E}} \widehat{U}_{1 Y} & =-\alpha \widehat{P}_{5 \mathrm{O} \xi}+\widehat{U}_{2 Y Y}+\widehat{U}_{1 Y} / r_{c}
\end{aligned}
$$

If we differentiate the second of these equations with respect to $Y$, use expressions (5.36), (5.42) for the velocity components, and change to the characteristic variable $\zeta$ defined in (5.41), we can simplify this system to

$$
\frac{\partial}{\partial \widehat{\xi}}\left(\widehat{U}_{5 \mathrm{OY}}\right)=\mp\left(\frac{2 \alpha \tau_{0}}{r_{c}}\right)^{1 / 2} \frac{\partial}{\partial \zeta}\left((\zeta-\mu \cos \xi)^{1 / 2} \kappa^{\prime}(\zeta)\right)
$$

where $\kappa(\zeta)$ is given by $(5.45)$, and $\partial / \partial \widehat{\xi}$ denotes differentiation with respect to $\xi$, holding $\zeta$ fixed.

To determine the velocity jump we write

$$
\widehat{U}_{5 \mathrm{O}}=\sum_{n=1}^{\infty} b_{n}(Y) \sin n \xi
$$

so that the quantity $\phi$ defined in $(5.11)$ is given by

$$
\phi=\lim _{Y \rightarrow \infty} b_{1}-\lim _{Y \rightarrow-\infty} b_{1}
$$

where

$$
b_{1}(Y)=\frac{1}{\pi} \int_{0}^{2 \pi} \widehat{U}_{5 \mathrm{O}}(\xi, Y) \sin \xi d \xi .
$$

By changing variables from $(\xi, Y)$ to $(\widehat{\xi}, \zeta)$, where $\xi=\widehat{\xi}$, integrating by parts with respect 
to $\widehat{\xi}$, and using (A 1), we finally obtain

$$
b_{1}=\frac{2}{\mu}\left(2 \zeta \kappa^{\prime}-\kappa\right)+\text { constant. }
$$

Then, using the properties of $\kappa$ given in (5.43), (5.45), (5.46) and (5.47), we can establish that

$$
\phi=\frac{2 \tau_{0} C_{1}}{\alpha r_{c}^{2} A_{0}^{1 / 2}},
$$

which is the result (5.52) quoted in the main text.

\section{REFERENCES}

Benney, D. J. \& Bergeron, R. F. 1969 A new class of nonlinear waves in parallel flows. Stud. Appl. Math. 48, 181-204.

Bodonyi, R. J., Smith, F. T. \& GajJar, J. 1983 Amplitude-dependent stability of boundary layer flow with a strongly nonlinear critical layer. IMA J. Appl. Math. 30, 1-19.

Brown, S. N. \& StewARTSON, K. 1978 The evolution of a small inviscid disturbance to a marginally unstable stratified shear flow; stage two. Proc. R. Soc. Lond. A 363, 174-194.

Cherhabili, A. \& Ehrenstein, U. 1995 Spatially localized two-dimensional equilibrium states in plane Couette flow. Eur. J. Mech. B/Fluids 14, 677-696.

Cipolla, K. M. \& Keith, W. L. 2003 High Reynolds number thick axisymmetric turbulent boundary layer measurements. Exp. Fluids 35, 477-485.

Clever R. M. \& Busse, F. H. 1997 Tertiary and quaternary solutions for plane Couette flow. J. Fluid Mech. 344, 137-153.

Cowley, S. J. \& Smith, F. T. 1985 On the stability of Poiseuille-Couette flow: a bifurcation of infinity. J. Fluid Mech. 156, 83-100.

DAvis, R. E. 1969 On the high Reynolds number flow over a wavy boundary. J. Fluid Mech. 36, 337-346.

Davies, S. J. \& White, C. M. 1928 An experimental study of the flow of water in pipes of rectangular section. Proc. Roy. Soc. A 119, 92-107.

Deguchi, K., Hall, P. \& Walton, A. G. 2013 The emergence of localized vortex-wave interaction states in plane Couette flow. J. Fluid Mech. (To appear).

Deguchi, K. \& Nagata, M. 2011 Bifurcations and instabilities in sliding Couette flow. J. Fluid Mech. 678, 156-178.

Ehrenstein, U., Nagata, M. \& Rincon, F. 2008 Two-dimensional nonlinear plane PoiseuilleCouette flow homotopy revisited. Phys. Fluids 20, 064103.

Frei, Ch., Lüscher, P \& Wintermantel, E. 2000 Thread-annular flow in vertical pipes. J. Fluid Mech. 410, 185-210.

Faisst, H. \& Eckhardt, B. 2003 Traveling waves in pipe flow. Phys. Rev. Lett. 91, 224502.

Gittler, Ph. 1993 Stability of Poiseuille-Couette flow between concentric cylinders. Acta Mechanica. 101, 1-13.

Haberman, R. 1972 Critical layers in parallel flows. Stud. Appl. Math. 51, 139-161.

Hall, P. \& Sherwin, S. 2010 Streamwise vortices in shear flows: harbingers of transition and the skeleton of coherent structures. J. Fluid Mech. 661, 178-205.

Hall, P. \& Smith, F. T. 1991 On strongly nonlinear vortex/wave interactions in boundarylayer transition. J. Fluid Mech. 227, 641-666.

Healey, J. J. 1995 On the neutral curve of the flat-plate boundary layer: comparison between experiment, Orr-Sommerfeld theory and asymptotic theory. J. Fluid Mech. 288, 59-73.

Henningson, D., Spalart, P. \& Kim, J. 1987 Numerical simulations of turbulent spots in plane Poiseuille and boundary-layer flow. Phys. Fluids 30(10), 2914-2917.

Itano, T. \& Toh, S. 2001 The dynamics of bursting process in wall turbulence. J. Phys. Soc. Jpn. 70, 703-716.

Joseph, D. D. \& CARmi, S. 1969 Stability of Poiseuille flow in pipes, annuli, and channels. Quart. Applied Math. 26, 575-599. 
Lemoult, G., Aider, J. \& Wesfreid. 2012 Experimental scaling law for the subcritical transition to turbulence in plane Poiseuille flow. Phys. Rev. E 85, 025303(R).

Menta, P. G. 2004 A unified well-posed computational approach for 2D Orr-Sommerfeld problem. J. Comput. Phys. 199(2), 541-557.

Menta, P. G. \& Healey, T. J. 2005 On steady solutions of symmetry-preserving perturbations of the two-dimensional Couette flow problem. Phys. Fluids 17, 094108.

NAGATA, M. 1990 Three-dimensional finite-amplitude solutions in plane Couette flow: bifurcation from infinity. J. Fluid Mech. 217, 519-527.

NishiOKA, M., IIDA, S. AND ICHIKAWA, Y. 1975 An experimental investigation of the stability of plane Couette flow. J. Fluid Mech. 72(4), 731-751.

Orszag, S. A. \& Kells, L. C. 1980 Transition to turbulence in plane Poiseuille and plane Couette flow. J. Fluid Mech. 72(4), 731-751.

Rincon, F. 2007 On the existence of two-dimensional nonlinear steady states in plane Couette flow. Phys. Fluids 19, 074105.

Schlichting, H. 1933 Laminare Strahlenausbreitung. Z. Angew Math. Mech. 13, 260-263.

Shands, J., Alfredsson, H. \& Lindgren, E. R. 1980 Annular pipe flow subject to axial motion of the inner boundary. Phis. Fluids. 23(10), 2144-2145.

SkufCA, J. D., Yorke, J. A. \& Eckhardt, B. 2006 Edge of chaos in a parallel shear flow. Phys. Rev. Lett. 96, 174101-174104.

Smith, F. T. \& Bodonyi R. J. 1982a Amplitude-dependent neutral modes in the HagenPoiseuille flow through a circular pipe. Proc. R. Soc. Lond. A 384, 463-489.

Smith, F. T. \& Bodonyi R. J. 1982b Nonlinear critical layers and their development in streaming-flow instability. J. Fluid Mech. 118, 165-185.

Tollmien, W. 1929 Uber die Entstehung der Turbulenz. Nachr. Ges. Wiss. Gottingen MathPyhs. Kl. II, 21-44.

Tsukahara, T., Seki, S., Kawamura, H. And Tochio, D. 2005 DNS of turbulent channel flow at very low Reynolds numbers. Proceedings of the Fourth International Symposium on Turbulence and Shear Flow Phenomena, 935-940.

Tutty, O. R. 2008 Flow along a long thin cylinder. J. Fluid Mech. 602, 1-37.

Waleffe, F. 1998 Three-dimensional coherent states in plane shear flows. Phys. Rev. Lett. 81(19), 4140-4143.

Walton, A. G. 2002 The temporal evolution of neutral modes in the impulsively started flow through a pipe of circular cross-section. J. Fluid Mech. 457, 339-376.

Walton, A. G. 2003 The nonlinear instability of thread-annular flow at high Reynolds number. J. Fluid Mech. 477, 227-257.

Walton, A. G. 2004 Stability of circular Poiseuille-Couette flow to axisymmetric disturbances. J. Fluid Mech. 500, 169-210.

Walton, A. G. 2005 The linear and nonlinear stability of thread-annular flow. Phil. Trans. R. Soc. A363, 1223-1233.

Walton, A. G. 2011 The stability of developing pipe flow at high Reynolds number and the existence of nonlinear neutral centre modes. J. Fluid Mech. 684, 284-315.

Wedin, H. \& Kerswell, R. R. 2004 Exact coherent structures in pipe flow: travelling wave solutions. J. Fluid Mech. 508, 333-371.

Wong, A. W. H. \& Walton, A. G. 2012 Axisymmetric travelling waves in annular CouettePoiseuille flow. Q. J. Mech. Appl. Math. 65, 293-311. 